$$
\begin{aligned}
& 10 \\
& \text { AVgebra \& } \\
& \text { Number } \\
& \text { Theory } \\
& \text { Volume } 5
\end{aligned}
$$

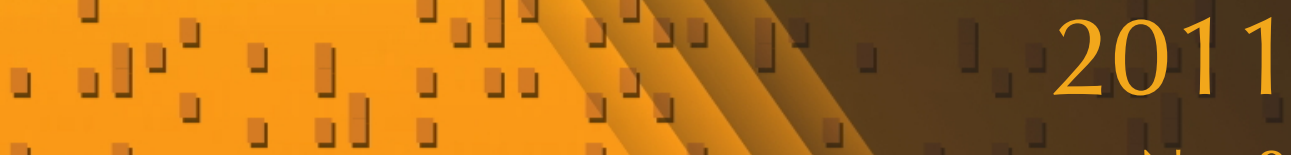

$$
\begin{aligned}
& \text { 」ป } \\
& \lrcorner
\end{aligned}
$$

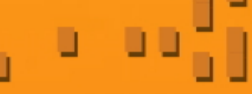

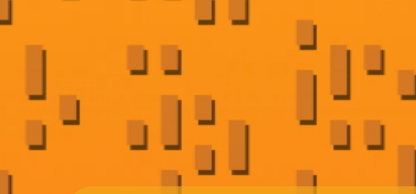

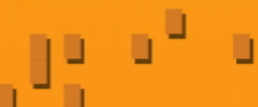

$$
\begin{aligned}
& \lrcorner \\
& \text { No. } 8
\end{aligned}
$$

Linear determinantal equations for all projective schemes

\lrcorner Jessica Sidman and Gregory G. Smith

」

\rfloor

.

Ј ل 」 


\title{
Linear determinantal equations for all projective schemes
}

\author{
Jessica Sidman and Gregory G. Smith
}

\begin{abstract}
We prove that every projective embedding of a connected scheme determined by the complete linear series of a sufficiently ample line bundle is defined by the $2 \times 2$ minors of a 1-generic matrix of linear forms. Extending the work of Eisenbud, Koh and Stillman for integral curves, we also provide effective descriptions for such determinantally presented ample line bundles on products of projective spaces, Gorenstein toric varieties, and smooth varieties.
\end{abstract}

\section{Introduction}

Relating the geometric properties of a variety to the structural features of its defining equations is a fundamental challenge in algebraic geometry. Describing generators for the homogeneous ideal associated to a projective scheme is a basic form of this problem. For a rational normal curve, a Segre variety, or a quadratic Veronese variety, the homogeneous ideal is conveniently expressed as the 2-minors (that is, the determinants of all $2 \times 2$ submatrices) of a generic Hankel matrix, a generic matrix, or a generic symmetric matrix respectively. These determinantal representations lead to a description of the minimal graded free resolution of the homogeneous ideal of the variety and equations for higher secant varieties. Mumford's "somewhat startling observation" [Mumford 1970, p. 31] is that a suitable multiple of every projective embedding is the intersection of a quadratic Veronese variety with a linear space and, hence, is defined by the 2-minors of a matrix of linear forms. Exercise 6.10 in [Eisenbud 2005] rephrases this as a "(vague) principle that embeddings of varieties by sufficiently positive bundles are often defined by ideals of $2 \times 2$ minors". Our primary goal is to provide a precise form of this principle.

Sidman was partially supported by NSF grant DMS-0600471 and the Clare Boothe Luce program. Smith was partially supported by NSERC and grant KAW 2005.0098 from the Knut and Alice Wallenberg Foundation.

MSC2000: primary 14A25; secondary 14F05, $13 \mathrm{D} 02$.

Keywords: determinantally presented, linear free resolution, Castelnuovo-Mumford regularity. 
To be more explicit, consider a scheme $X$ embedded in $\mathbb{P}^{r}$ by the complete linear series of a line bundle $L$. As in [Eisenbud et al. 1988, p. 514], the line bundle $L$

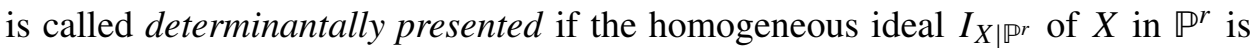
generated by the 2-minors of a 1-generic matrix (that is, no conjugate matrix has a zero entry) of linear forms. Definition 3.1 in [Green 1984b] states that a property holds for a sufficiently ample line bundle on $X$ if there exists a line bundle $A$ such that the property holds for all $L \in \operatorname{Pic}(X)$ for which $L \otimes A^{-1}$ is ample. Our main result is this:

Theorem 1.1. Every sufficiently ample line bundle on a connected scheme is determinantally presented.

We also describe, in terms of Castelnuovo-Mumford regularity, a set of determinantally presented line bundles on an arbitrary projective scheme; see Corollary 3.3.

This theorem is a new incarnation of a well-known phenomenon - roughly speaking, the complexity of the first few syzygies of a projective subscheme is inversely related to the positivity of the corresponding linear series. Nevertheless, Theorem 1.1 counterintuitively implies that most projective embeddings by a complete linear series are simply the intersection of a Segre variety with a linear subspace. More precisely, if we fix the Euclidean metric on the ample cone $\operatorname{Amp}(X)$ that it inherits from the finite-dimensional real vector space $N^{1}(X) \otimes \mathbb{R}$, then the fraction of determinantally presented ample classes within distance $\rho$ of the trivial class approaches 1 has $\rho$ tends to $\infty$.

Theorem 1.1 also has consequences beyond showing that the homogeneous ideal is generated by quadrics of rank at least 2. Proposition 6.13 in [Eisenbud 2005] shows that an Eagon-Northcott complex is a direct summand of the minimal graded free resolution of the ideal. Despite the classic examples, being able to give a complete description of this resolution in the general setting seems overly optimistic. However, a determinantal presentation provides many equations for higher secant varieties; see [Eisenbud et al. 1988, Proposition 1.3]. For a scheme $X \subset \mathbb{P}^{r}$, let $\operatorname{Sec}^{k}(X)$ be the Zariski closure of the union of the linear spaces spanned by collections of $k+1$ points on $X$. A natural generalization of Theorem 1.1 would be as follows:

Conjecture 1.2. Let $k$ be a positive integer. If $X \subset \mathbb{P}^{r}$ is embedded by the complete linear series of a sufficiently ample line bundle, then the homogeneous ideal of $\operatorname{Sec}^{k}(X)$ is generated by the $(k+2)$-minors of a 1-generic matrix of linear forms.

This conjecture holds for rational normal curves [Eisenbud 1988, Proposition 4.3], rational normal scrolls [Catalano-Johnson 1996, Proposition 2.2], Segre varieties, and quadratic Veronese varieties [Sturmfels and Sullivant 2006, Section 4]. It also extends the conjecture for curves appearing in [Eisenbud et al. 1988, p. 518] for which [Ravi 1994] proves a set-theoretic version and for which [Ginensky 
2010, Section 7] proves a scheme-theoretic version. Although Theorem 1.1.4 in [Buczyński et al. 2010] produces counterexamples to this conjecture for some singular $X$, Corollary 1.2.4 therein provides supporting evidence when $X$ is smooth. Theorem 1.3 in [Buczyński and Buczyński 2010] suggests that the secant varieties in Conjecture 1.2 should be replaced by cactus varieties.

The secondary goal of this article is to effectively bound the determinantally presented line bundles on specific schemes. For an integral curve of genus $g$, Theorem 1 in [Eisenbud et al. 1988] shows that a line bundle is determinantally presented when its degree is at least $4 g+2$ and this bound is sharp. We provide the analogous result on smooth varieties and Gorenstein toric varieties:

Theorem 1.3. Let $X$ be a smooth variety of dimension $n$ or an $n$-dimensional Gorenstein toric variety and let $A$ be a very ample line bundle on $X$ such that $(X, A) \neq\left(\mathbb{P}^{n}, \mathbb{O}_{\mathbb{P}}^{n}(1)\right)$. If $B$ is a nef line bundle, $K_{X}$ is the dualizing bundle on $X$, and $L:=K_{X}^{2} \otimes A^{j} \otimes B$ with $j \geqslant 2 n+2$, then $L$ is determinantally presented.

As an application of our methods, we describe determinantally presented ample line bundles on products of projective spaces; see Theorem 4.1.

To prove these theorems, we need a source of appropriate matrices. Composition of linear series (also known as multiplication in the total coordinate ring or the Cox ring) traditionally supply the required matrices. If $X \subset \mathbb{P}^{r}$ is embedded by the complete linear series for a line bundle $L$, then $H^{0}(X, L)$ is the space of linear forms on $\mathbb{P}^{r}$. Factoring $L$ as $L=E \otimes E^{\prime}$ for some $E, E^{\prime} \in \operatorname{Pic}(X)$ yields a natural map

$$
\mu: H^{0}(X, E) \otimes H^{0}\left(X, E^{\prime}\right) \rightarrow H^{0}\left(X, E \otimes E^{\prime}\right)=H^{0}(X, L) .
$$

By choosing ordered bases $y_{1}, \ldots, y_{s} \in H^{0}(X, E)$ and $z_{1}, \ldots, z_{t} \in H^{0}\left(X, E^{\prime}\right)$, we obtain an associated $(s \times t)$-matrix $\Omega:=\left[\mu\left(y_{i} \otimes z_{j}\right)\right]$ of linear forms. The matrix $\Omega$ is 1-generic and its ideal $\mathrm{I}_{2}(\Omega)$ of 2-minors vanishes on $X$; see [Eisenbud 2005, Proposition 6.10]. Numerous classical examples of this construction can be found in [Room 1938].

With these preliminaries, the problem reduces to finding conditions on $E$ and $E^{\prime}$ that guarantee that $I_{X \mid \mathbb{P} r}=\mathrm{I}_{2}(\Omega)$. Inspired by the approach in [Eisenbud et al. 1988], Theorem 3.2 achieves this by placing restrictions on certain modules arising from the line bundles $L, E$, and $E^{\prime}$. The key hypotheses require these modules to have a linear free presentation; the generators of the $\mathbb{N}$-graded modules have degree 0 and their first syzygies must have degree 1. Methods introduced by Green and Lazarsfeld [Green 1984a; Green and Lazarsfeld 1985] — for an expository account see [Eisenbud 2005, Section 8; Green 1989; Lazarsfeld 1989, Section1] — yield a cohomological criterion for our modules to have a linear free presentation. Hence, we can prove Theorem 1.1 by combining this with uniform vanishing results derived from Castelnuovo-Mumford regularity. Building on known conditions (that 
is, sufficient conditions for a line bundle to satisfy $N_{1}$ ), we obtain effective criteria for the appropriate modules to have a linear free presentation on Gorenstein toric varieties, and smooth varieties.

Rather than focusing exclusively on a single factorization of the line bundle $L$, we set up the apparatus to handle multiple factorizations; see Lemma 3.1. Multiple factorizations of a line bundle were used in [Graf v. Bothmer and Hulek 2004] to study the equations and syzygies of elliptic normal curves and their secant varieties. They also provide a geometric interpretation for the flattenings appearing in [Garcia et al. 2005, Section 7] and [Catalisano et al. 2008, p. 1915]. Using this more general setup, we are able to describe the homogeneous ideal for every embedding of a product of projective spaces by a very ample line bundle as the 2-minors of appropriate 1-generic matrices of linear forms; see Proposition 4.4.

Conventions. In this paper, $\mathbb{N}$ is the set of nonnegative integers, $\mathbb{1}_{W} \in \operatorname{Hom}(W, W)$ is the identity map, and $\mathbf{1}:=(1, \ldots, 1)$ is the vector in which every entry is 1 . We work over an algebraically closed field $k$ of characteristic zero. A variety is always irreducible and all of our toric varieties are normal. For a vector bundle $U$, we write $U^{j}$ for the $j$-fold tensor product $U^{\otimes j}=U \otimes \cdots \otimes U$.

\section{Linear free presentations}

This section collects the criteria needed to show that certain modules arising from line bundles have a linear free presentation. While accomplishing this, we also establish some notation and nomenclature used throughout the document.

Let $X$ be a projective scheme over $\mathbb{k}$, let $\mathscr{F}_{F}$ be a coherent $O_{X}$-module, and let $L$ be a line bundle on $X$. We write $\Gamma(L):=H^{0}(X, L)$ for the $\mathrm{k}$-vector space of global sections and $S:=\operatorname{Sym}(\Gamma(L))$ for the homogeneous coordinate ring of $\mathbb{P}^{r}:=\mathbb{P}(\Gamma(L))$. Consider the $\mathbb{N}$-graded $S$-module $F:=\bigoplus_{j \geqslant 0} H^{0}\left(X, \mathscr{F} \otimes L^{j}\right)$. When $\mathscr{F}_{F}=\mathrm{O}_{X}, F$ is the section ring of $L$. However, when $\mathscr{F}=L$, the module $F$ is the truncation of the section ring omitting the zeroth graded piece and shifting degrees by -1 . Let $P_{\bullet}$ be a minimal graded free resolution of $F$ :

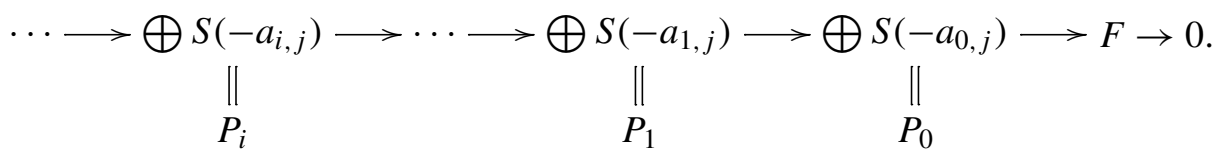

Following [Eisenbud et al. 1988, p. 515], we say that, for $p \in \mathbb{N}$, F F has a linear free resolution to stage $p$ with respect to $L$ or $F$ has a linear free resolution to stage $p$ if $P_{i}=\bigoplus S(-i)$ for all $0 \leqslant i \leqslant p$. Thus, $F$ has a linear free resolution to stage 0 if and only if it is generated in degree 0 . Since having a linear free resolution to stage 1 implies that the relations among the generators (also known as first syzygies) are linear, the module $F$ has a linear free resolution to stage 1 if and only if it has a 
linear free presentation. In this case, we say that $\mathscr{F}$ has a linear free presentation with respect to $L$. More generally, having a linear free resolution to stage $p$ is the module-theoretic analogue of the $N_{p}$-property introduced in [Green and Lazarsfeld 1985, Section 3]. If $X$ is connected, then the line bundle $L$ satisfies $N_{1}$ precisely when $L$ has a linear free presentation with respect to itself and satisfies $N_{p}$ when $L$ has a linear free resolution to stage $p$. Following [Ein and Lazarsfeld 1993, Convention 0.4], we do not assume that $X$ is normal.

Henceforth, we assume that $L$ is globally generated. In other words, the natural evaluation map ev $\operatorname{ev}_{L}: \Gamma(L) \otimes_{\mathbb{k}} \mathrm{O}_{X} \rightarrow L$ is surjective. If $M_{L}:=\operatorname{Ker}\left(\mathrm{ev}_{L}\right)$, then $M_{L}$ is a vector bundle of rank $r:=\operatorname{dim}_{\mathbb{k}} \Gamma(L)-1$ that sits in the short exact sequence

$$
0 \rightarrow M_{L} \longrightarrow \Gamma(L) \otimes_{\mathbb{k}} \mathrm{O}_{X} \longrightarrow L \rightarrow 0 .
$$

For convenience, we record the following cohomological criteria, which is a minor variant of [Eisenbud 2005, Theorem 5.6], [Green 1989, Proposition 2.4], or [Ein and Lazarsfeld 1993, Lemma 1.6].

Lemma 2.1. If $H^{1}\left(X, \bigwedge^{i} M_{L} \otimes \mathscr{F} \otimes L^{j}\right)=0$ for all $1 \leqslant i \leqslant p+1$ and all $j \geqslant 0$, then the coherent $\mathrm{O}_{X}$-module $\mathscr{F}$ has a linear free resolution to stage $p$ with respect to $L$. In characteristic zero, $\bigwedge^{i} M_{L}$ is a direct summand of $M_{L}^{i}$, so it suffices to show $H^{1}\left(X, M_{L}^{i} \otimes \mathscr{F} \otimes L^{j}\right)=0$ for all $1 \leqslant i \leqslant p+1$ and all $j \geqslant 0$.

Sketch of proof. The key observation is that the graded Betti numbers for the minimal free resolution of $F$ can be computed via Koszul cohomology. If $L$ is globally generated and $\mathbb{P}^{r}=\mathbb{P}\left(H^{0}(X, L)\right)$, then there is a morphism $\varphi_{L}: X \rightarrow \mathbb{P}^{r}$ with $\varphi_{L}^{*}\left(\mathscr{O}_{\mathbb{p} r}(1)\right)=L$. Since the pullback by $\varphi_{L}^{*}$ of $0 \rightarrow M_{\mathscr{C}_{\mathbb{p}}(1)} \rightarrow \Gamma\left(\mathcal{O}_{\mathbb{p} r}(1)\right) \otimes_{\mathbb{k}}$ $\mathcal{O}_{\mathbb{p} r} \rightarrow \mathcal{O}_{\mathbb{P}}(1) \rightarrow 0$ is just $(*)$, the proof of [Eisenbud 2005, Theorem 5.6] goes through working on $X$ instead of $\mathbb{P}^{r}$.

Multigraded Castelnuovo-Mumford regularity, as developed in [Maclagan and Smith 2004, Section 6] or [Hering et al. 2006, Section 2], allows us to exploit this criteria. To be more precise, fix a list $B_{1}, \ldots, B_{\ell}$ of globally generated line bundles on $X$. For a vector $\boldsymbol{u}:=\left(u_{1}, \ldots, u_{\ell}\right) \in \mathbb{Z}^{\ell}$, we set $\boldsymbol{B}^{\boldsymbol{u}}:=B_{1}^{u_{1}} \otimes \cdots \otimes B_{\ell}^{u_{\ell}}$ and we write $\mathfrak{B}:=\left\{\boldsymbol{B}^{\boldsymbol{u}}: \boldsymbol{u} \in \mathbb{N}^{\ell}\right\} \subset \operatorname{Pic}(X)$ for the submonoid generated by these line bundles. If $\boldsymbol{e}_{1}, \ldots, \boldsymbol{e}_{\ell}$ is the standard basis for $\mathbb{Z}^{\ell}$ then $\boldsymbol{B}^{\boldsymbol{e}_{j}}=B_{j}$. A coherent $\mathcal{O}_{X^{-}}$ module $\mathscr{F}_{F}$ is said to be regular with respect to $B_{1}, \ldots, B_{\ell}$ if $H^{i}\left(X, \mathscr{F}_{F} \otimes \boldsymbol{B}^{-\boldsymbol{u}}\right)=0$ for all $i>0$ and all $\boldsymbol{u} \in \mathbb{N}^{\ell}$ satisfying $|\boldsymbol{u}|:=u_{1}+\cdots+u_{\ell}=i$. When $\ell=1$, we recover the version of Castelnuovo-Mumford regularity found in [Lazarsfeld 2004, Section 1.8].

Although the definition may not be intuitive, the next result shows that regular line bundles are at least ubiquitous. 
Lemma 2.2. Let $X$ be a scheme and let $B_{1}, \ldots, B_{\ell}$ be globally generated line bundles on $X$. If there is a positive vector $\boldsymbol{w} \in \mathbb{Z}^{\ell}$ such that $\boldsymbol{B}^{w}$ is ample, then a sufficiently ample line bundle on $X$ is regular with respect to $B_{1}, \ldots, B_{\ell}$.

The hypothesis on $w$ means that the cone $\operatorname{pos}\left(B_{1}, \ldots, B_{\ell}\right)$ generated by $B_{1}, \ldots, B_{\ell}$ contains an ample line bundle. In other words, the subcone $\operatorname{pos}\left(B_{1}, \ldots, B_{\ell}\right)$ of $\operatorname{Nef}(X)$ has a nonempty intersection with the interior of $\operatorname{Nef}(X)$.

Proof. It suffices to find a line bundle $A$ on $X$ such that, for any nef line bundle $C$, $A \otimes C$ is regular with respect to $B_{1}, \ldots, B_{\ell}$. Because $\boldsymbol{B}^{w}$ is ample, Fujita's vanishing theorem (for example, [Fujita 1983, Theorem 1]) implies that there is $k \in \mathbb{N}$ such that, for any nef line bundle $C$, we have $H^{i}\left(X, \boldsymbol{B}^{j w} \otimes C\right)=0$ for all $i>0$ and all $j \geqslant k$. Let $n:=\operatorname{dim} X$ and consider $A:=\boldsymbol{B}^{(k+n) \boldsymbol{w}}$. Since $\boldsymbol{w}$ is positive, the line bundle $\boldsymbol{B}^{n \boldsymbol{w}-\boldsymbol{u}}$ is nef for all $\boldsymbol{u} \in \mathbb{N}^{\ell}$ with $0 \leqslant|\boldsymbol{u}| \leqslant n$. Therefore, we have $H^{i}\left(X,(A \otimes C) \otimes \boldsymbol{B}^{-u}\right)=H^{i}\left(X, \boldsymbol{B}^{k \boldsymbol{w}} \otimes\left(\boldsymbol{B}^{n \boldsymbol{w}-\boldsymbol{u}} \otimes C\right)\right)=0$ for all $i>0$ and all $\boldsymbol{u} \in \mathbb{N}^{\ell}$ satisfying $|\boldsymbol{u}|=i$.

Before describing the pivotal results in this section, we record a technical lemma bounding the regularity of certain tensor products. Our approach is a hybrid of [Lazarsfeld 2004, Proposition 1.8.9 and Remark 1.8.16].

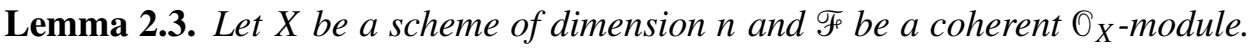
Fix a vector bundle $V$ and a globally generated ample line bundle $B$ on $X$. If $m$ is positive integer such that $\mathscr{F}, V$, and $B^{m}$ are all regular with respect to $B$, then $\mathscr{F} \otimes V \otimes B^{w}$ is also regular with respect to $B$ for all $w \geqslant(m-1)(n-1)$.

Proof. Since $\mathscr{F}$ and $B^{m}$ are regular with respect to $B$, either [Arapura 2004, Corollary 3.2] or [Maclagan and Smith 2004, Theorem 7.8] (also compare with [Lazarsfeld 2004, Proposition 1.8.8]) produces a locally free resolution of $\mathscr{F}$ of the form

$$
\cdots \longrightarrow \oplus B^{-j m} \longrightarrow \cdots \longrightarrow \oplus B^{-m} \longrightarrow \oplus \mathrm{O}_{X} \longrightarrow \mathscr{F}_{F} \rightarrow 0 .
$$

Tensoring by a locally free sheaf preserves exactness, so we get the exact complex

$$
\cdots \longrightarrow \oplus V \otimes B^{w-j m} \longrightarrow \cdots \longrightarrow \bigoplus V \otimes B^{w} \longrightarrow \mathscr{F} \otimes V \otimes B^{w} \rightarrow 0 .
$$

Since $V$ is also regular with respect to $B$, Mumford's lemma (see for example [Lazarsfeld 2004, Theorem 1.8.5]) implies that $H^{i+j}\left(X, V \otimes B^{w-j m-i}\right)=0$ for $i \geqslant 1$ provided we have $w-j m-i \geqslant-i-j$. Chasing through the complex (see [Lazarsfeld 2004, Proposition B.1.2]), we conclude that $\mathscr{F} \otimes V \otimes B^{w}$ is also regular with respect to $B$ when $w \geqslant(m-1)(n-1)$.

The next three propositions each provide sufficient conditions for an appropriate line bundle to have a linear free presentation with respect to another line bundle. 
Proposition 2.4. Fix a positive integer $m$ and a scheme $X$ of dimension $n$. Let $L$ be a line bundle on $X$ and let $B$ be a globally generated ample line bundle on $X$. If $L^{j}$ and $B^{m}$ are regular with respect to $B$ for all $j \geqslant 1$, then $B^{w}$ has a linear free presentation with respect to $L$ for all $w \geqslant 2(m-1) n+1$.

Proof. We first prove that $M_{L} \otimes B^{m}$ is regular with respect to $B$. Tensoring (*) with $B^{m-i}$ and taking the associated long exact sequence gives

$\Gamma(L) \otimes H^{0}\left(X, B^{m-i}\right) \longrightarrow H^{0}\left(X, L \otimes B^{m-i}\right) \longrightarrow H^{1}\left(X, M_{L} \otimes B^{m-i}\right) \longrightarrow \cdots$ $\longrightarrow H^{i-1}\left(X, L \otimes B^{m-i}\right) \longrightarrow H^{i}\left(X, M_{L} \otimes B^{m-i}\right) \longrightarrow \Gamma(L) \otimes H^{i}\left(X, B^{m-i}\right)$.

Since $L$ is regular with respect to $B$, Mumford's lemma shows that, for all $k \in \mathbb{N}$, the map $\Gamma(L) \otimes H^{0}\left(X, B^{k}\right) \rightarrow H^{0}\left(X, L \otimes B^{k}\right)$ is surjective and, for all $i>0$ and all $k \in \mathbb{N}$, we have $H^{i}\left(X, L \otimes B^{k-i}\right)=0$. As $m$ is a positive integer, the map $\Gamma(L) \otimes H^{0}\left(X, B^{m-1}\right) \rightarrow H^{0}\left(X, L \otimes B^{m-1}\right)$ is surjective and $H^{i-1}\left(X, L \otimes B^{m-i}\right)=$ 0 for all $i>1$. Since $B^{m}$ is also regular with respect to $B$, we have $H^{i}\left(X, B^{m-i}\right)=0$ for all $i>0$. It follows that $H^{i}\left(X, M_{L} \otimes B^{m-i}\right)=0$ for all $i>0$.

By Lemma 2.1, it suffices to show that

$$
H^{1}\left(X, M_{L} \otimes B^{w} \otimes L^{j}\right)=0 \quad \text { and } \quad H^{1}\left(X, M_{L}^{2} \otimes B^{w} \otimes L^{j}\right)=0
$$

for all $j \in \mathbb{N}$. Thus, it suffices to show that the vector bundles $M_{L} \otimes B^{w+1} \otimes L^{j}$ and $M_{L}^{2} \otimes B^{w+1} \otimes L^{j}$ are both regular with respect to $B$. If $w \geqslant(m-1) n$, then Lemma 2.3 implies that $\left(M_{L} \otimes B^{m}\right) \otimes L^{j} \otimes B^{w+1-m}=M_{L} \otimes B^{w+1} \otimes L^{j}$ is regular with respect to $B$. Similarly, if $w \geqslant 2(m-1) n+1$, then using Lemma 2.3 twice establishes that the vector bundle

$\left(\left(M_{L} \otimes B^{m}\right) \otimes\left(M_{L} \otimes B^{m}\right) \otimes B^{(m-1)(n-1)}\right) \otimes L^{j} \otimes B^{w-m n-m+n}=M_{L}^{2} \otimes B^{w+1} \otimes L^{j}$ is also regular with respect to $B$.

By adapting the proof of [Hering et al. 2006, Theorem 1.1], we obtain the second proposition.

Proposition 2.5. Let $\boldsymbol{m} \in \mathbb{N}^{\ell}$ be a vector satisfying $\boldsymbol{B}^{\boldsymbol{m}-\boldsymbol{e}_{j}} \in \mathfrak{B}$ for all $1 \leqslant j \leqslant \ell$ and let the coherent $\mathrm{O}_{X}$-module $\mathscr{F}_{\boldsymbol{F}}$ be regular with respect to $B_{1}, \ldots, B_{\ell}$. If $L:=\boldsymbol{B}^{\boldsymbol{m}}$ and the map

$$
\Gamma(L) \otimes H^{0}\left(X, \mathscr{F} \otimes \boldsymbol{B}^{-\boldsymbol{e}_{j}}\right) \rightarrow H^{0}\left(X, \mathscr{F}_{\mathbb{F}} \otimes \boldsymbol{B}^{\boldsymbol{m}-\boldsymbol{e}_{j}}\right)
$$

is surjective for all $1 \leqslant j \leqslant \ell$, then $\mathscr{F}$ has a linear presentation with respect to $L$.

The condition that $\boldsymbol{B}^{\boldsymbol{m}-\boldsymbol{e}_{j}} \in \mathfrak{B}$ for all $1 \leqslant j \leqslant \ell$ implies that $L=\boldsymbol{B}^{\boldsymbol{m}}$ lies in the interior of the cone $\operatorname{pos}\left(B_{1}, \ldots, B_{\ell}\right)$. 
Proof. We first prove that $M_{L} \otimes \mathscr{F}$ is regular with respect to $B_{1}, \ldots, B_{\ell}$. Tensoring $(*)$ with $\mathscr{F} \otimes \boldsymbol{B}^{-\boldsymbol{u}}$ and taking the associated long exact sequence gives

$\Gamma(L) \otimes H^{0}\left(X, \mathscr{F} \otimes \boldsymbol{B}^{-\boldsymbol{u}}\right) \longrightarrow H^{0}\left(X, \mathscr{F} \otimes \boldsymbol{B}^{\boldsymbol{m}-\boldsymbol{u}}\right)$

$\longrightarrow H^{1}\left(X, M_{L} \otimes \mathscr{F} \otimes \boldsymbol{B}^{-\boldsymbol{u}}\right) \longrightarrow \cdots \longrightarrow H^{i-1}\left(X, \mathscr{F} \otimes \boldsymbol{B}^{\boldsymbol{m}-\boldsymbol{u}}\right)$

$\longrightarrow H^{i}\left(X, M_{L} \otimes \mathscr{F} \otimes \boldsymbol{B}^{-\boldsymbol{u}}\right) \longrightarrow \Gamma(L) \otimes H^{i}\left(X, \mathscr{F} \otimes \boldsymbol{B}^{-\boldsymbol{u}}\right)$.

Since $\mathscr{F}$ is regular with respect to $B_{1}, \ldots, B_{\ell}$, Theorem 2.1 in [Hering et al. 2006] shows that, for all $i>0$ and all $\boldsymbol{u}, \boldsymbol{v} \in \mathbb{N}^{\ell}$ with $|\boldsymbol{u}|=i, H^{i}\left(X, \mathscr{F} \otimes \boldsymbol{B}^{\boldsymbol{v}-\boldsymbol{u}}\right)=0$. As $\boldsymbol{B}^{\boldsymbol{m}-\boldsymbol{e}_{j}} \in \mathfrak{B}$ for $1 \leqslant j \leqslant \ell$, we see that $H^{i-1}\left(X, \mathscr{F} \otimes \boldsymbol{B}^{\boldsymbol{m}-\boldsymbol{u}}\right)=0$ for all $i>1$ and all $\boldsymbol{u} \in \mathbb{N}^{\ell}$ satisfying $|\boldsymbol{u}|=i$. By hypothesis, the map

$$
\Gamma(L) \otimes H^{0}\left(X, \mathscr{F} \otimes \boldsymbol{B}^{-\boldsymbol{e}_{j}}\right) \rightarrow H^{0}\left(X, \mathscr{F} \otimes \boldsymbol{B}^{\boldsymbol{m}-\boldsymbol{e}_{j}}\right)
$$

is surjective for all $1 \leqslant j \leqslant \ell$. It follows that $H^{i}\left(X, M_{L} \otimes \mathscr{F} \otimes \boldsymbol{B}^{-\boldsymbol{u}}\right)=0$ for all $i>0$ and all $\boldsymbol{u} \in \mathbb{N}^{\ell}$ such that $|\boldsymbol{u}|=i$.

By Lemma 2.1, it suffices to show that

$$
H^{1}\left(X, M_{L} \otimes \mathscr{F} \otimes L^{j}\right) \quad \text { and } \quad H^{1}\left(X, M_{L}^{2} \otimes \mathscr{F} \otimes L^{j}\right)
$$

are zero for $j \in \mathbb{N}$. Since $M_{L} \otimes F_{F}$ is regular with respect to $B_{1}, \ldots, B_{\ell}$, the vanishing of the first group follows from Theorem 2.1 [ibid.]. For the second, tensoring (*) with $M_{L} \otimes \mathscr{F} \otimes L^{j}$ gives the exact sequence

$\Gamma(L) \otimes H^{0}\left(X, M_{L} \otimes \mathscr{F} \otimes L^{j}\right) \longrightarrow H^{0}\left(X, M_{L} \otimes \mathscr{F} \otimes L^{j+1}\right)$

$$
\longrightarrow H^{1}\left(X, M_{L}^{2} \otimes \mathscr{F} \otimes L^{j}\right) \rightarrow 0 .
$$

Because $M_{L} \otimes \mathscr{F}$ is regular with respect to $B_{1}, \ldots, B_{\ell}$, Theorem 2.1 [ibid.] also shows that the left map is surjective for all $j \geqslant 0$.

Our third proposition is a variant of [Ein and Lazarsfeld 1993, Proposition 3.1].

Proposition 2.6. Let $X$ be a smooth variety of dimension $n$, let $K_{X}$ be its canonical bundle, and let $A$ be a very ample line bundle on $X$ such that $(X, A) \neq$ $\left(\mathbb{P}^{n}, \mathcal{O}_{\mathbb{P} n}(1)\right)$. Suppose that $B$ and $C$ are nef line bundles on $X$. If the integers $w$ and $m$ are both greater than $n$, then the line bundle $K_{X} \otimes A^{w} \otimes B$ has a linear free presentation with respect to $K_{X} \otimes A^{m} \otimes C$.

Proof. Let $\mathscr{F}:=K_{X} \otimes A^{w} \otimes B$ and $L:=K_{X} \otimes A^{m} \otimes C$. Since [Ein and Lazarsfeld 1993, Proposition 3.1] shows that $L$ satisfies $N_{0}$ and [Ein and Lazarsfeld 1993, Equation 3.2] shows that $H^{1}\left(X, M_{L}^{i} \otimes \mathscr{F} \otimes L^{j}\right)=0$ for all $1 \leqslant i \leqslant 2$ and all $j \geqslant 0$, Lemma 2.1 completes the proof. 


\section{Determinantally presented line bundles}

The goal of this section is to prove Theorem 1.1. We realize this goal by developing general methods for showing that a line bundle is determinantally presented; see Theorem 3.2.

Suppose $X \subset \mathbb{P}^{r}$ is embedded by the complete linear series for a line bundle $L$. Factor $L$ as $L=E \otimes E^{\prime}$ for some $E, E^{\prime} \in \operatorname{Pic}(X)$ and denote by

$$
\mu_{E, E^{\prime}}: H^{0}(X, E) \otimes H^{0}\left(X, E^{\prime}\right) \rightarrow H^{0}(X, L)
$$

the natural multiplication map. Choose ordered bases $y_{1}, \ldots, y_{s}$ and $z_{1}, \ldots, z_{t}$ for the $\mathrm{k}$-vector spaces $H^{0}(X, E)$ and $H^{0}\left(X, E^{\prime}\right)$, respectively. Define $\Omega=\Omega\left(E, E^{\prime}\right)$ to be the associated $(s \times t)$-matrix $\left[\mu_{E, E^{\prime}}\left(y_{i} \otimes z_{j}\right)\right]$ of linear forms. Its ideal $\mathrm{I}_{2}(\Omega)$ of 2-minors is independent of the choice of bases. Then [Eisenbud 2005, Proposition 6.10] shows that $\Omega$ is 1-generic and that $\mathrm{I}_{2}(\Omega)$ vanishes on $X$.

Our key technical result is inspired by [Eisenbud et al. 1988, Section 2].

Lemma 3.1. If $L$ is a very ample line bundle on $X$ satisfying $N_{1}$ and $\left\{\left(E_{i}, E_{i}^{\prime}\right)\right\}$ is a family of factorizations for $L$, then the commutative diagram ( $W$ has exact rows and columns. Moreover, if $\varphi_{2}$ is surjective, then the homogeneous ideal $I_{X \mid \mathbb{P} r}$ is generated by the 2-minors of the matrices $\Omega\left(E_{i}, E_{i}^{\prime}\right)$ if and only if $Q_{2}$ surjects onto $Q_{1}$.

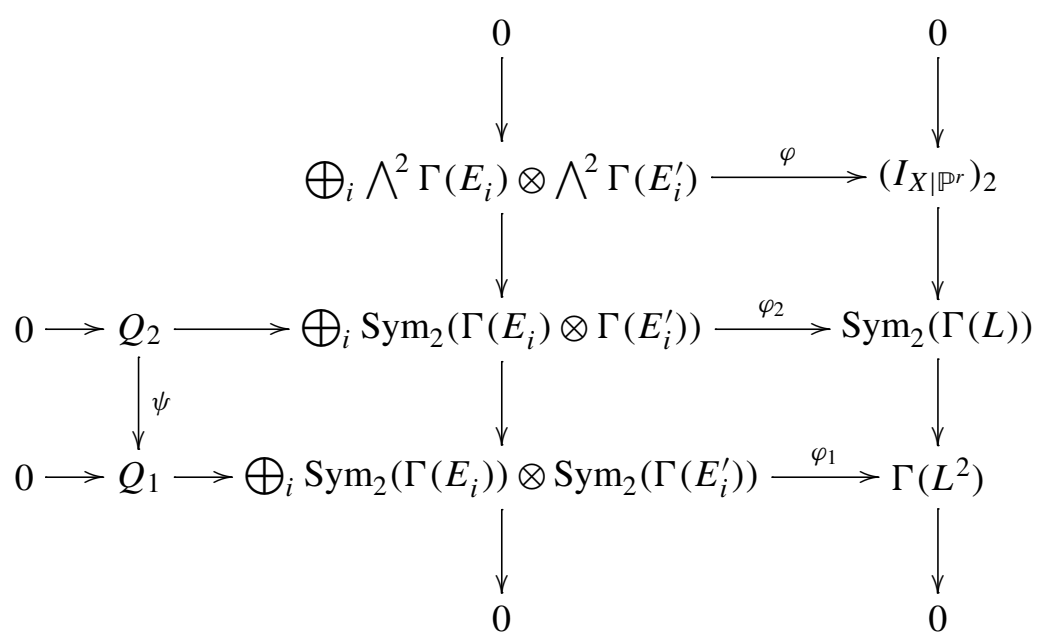

Proof. To begin, we prove the columns are exact. Since $L$ satisfies $N_{0}$ (that is, the natural maps $\operatorname{Sym}_{j}(\Gamma(L)) \rightarrow H^{0}\left(X, L^{j}\right)$ are surjective for all $\left.j \in \mathbb{N}\right)$, the ideal $I_{X \mid \mathbb{P}^{r}}$ is the kernel of the map from the homogeneous coordinate ring of $\mathbb{P}^{r}$ to the section ring of $L$. By taking the quadratic components, we obtain the right column. 
The middle column is the direct sum of the complexes:

$0 \rightarrow \bigwedge^{2} \Gamma\left(E_{i}\right) \otimes \bigwedge^{2} \Gamma\left(E_{i}^{\prime}\right) \longrightarrow \operatorname{Sym}_{2}\left(\Gamma\left(E_{i}\right) \otimes \Gamma\left(E_{i}^{\prime}\right)\right)$

$$
\longrightarrow \operatorname{Sym}_{2}\left(\Gamma\left(E_{i}\right)\right) \otimes \operatorname{Sym}_{2}\left(\Gamma\left(E_{i}^{\prime}\right)\right) \rightarrow 0 .
$$

The map $\bigwedge^{2} \Gamma\left(E_{i}\right) \otimes \bigwedge^{2} \Gamma\left(E_{i}^{\prime}\right) \rightarrow \operatorname{Sym}_{2}\left(\Gamma\left(E_{i}\right) \otimes \Gamma\left(E_{i}^{\prime}\right)\right)$, defined by

$$
e \wedge f \otimes e^{\prime} \wedge f^{\prime} \mapsto\left(e \otimes e^{\prime}\right) \cdot\left(f \otimes f^{\prime}\right)-\left(e \otimes f^{\prime}\right) \cdot\left(f \otimes e^{\prime}\right),
$$

is simply the inclusion map determined by the 2-minors of the generic matrix. The $\operatorname{map} \operatorname{Sym}_{2}\left(\Gamma\left(E_{i}\right) \otimes \Gamma\left(E_{i}^{\prime}\right)\right) \rightarrow \operatorname{Sym}_{2}\left(\Gamma\left(E_{i}\right)\right) \otimes \operatorname{Sym}_{2}\left(\Gamma\left(E_{i}^{\prime}\right)\right)$ is $\left(e \otimes e^{\prime}\right) \cdot\left(f \otimes f^{\prime}\right) \mapsto$ ef $\otimes e^{\prime} f^{\prime}$. Hence, each of these complexes is exact, so the middle column also is. By definition, $Q_{1}$ and $Q_{2}$ are the kernels of the $\varphi_{1}$ and $\varphi_{2}$ respectively, and $\psi$ is the induced map between them.

We next identify the horizontal maps. By applying the functor $\operatorname{Sym}_{2}$ to $\mu_{E_{i}, E_{i}^{\prime}}$, we obtain a map from $\operatorname{Sym}_{2}\left(\Gamma\left(E_{i}\right) \otimes \Gamma\left(E_{i}^{\prime}\right)\right)$ to $\operatorname{Sym}_{2}(\Gamma(L))$ for each $i$, and $\varphi_{2}$ is their direct sum. The composite map

$$
\mu_{L, L} \circ\left(\mu_{E_{i}, E_{i}^{\prime}} \otimes \mu_{E_{i}, E_{i}^{\prime}}\right): \Gamma\left(E_{i}\right) \otimes \Gamma\left(E_{i}^{\prime}\right) \otimes \Gamma\left(E_{i}\right) \otimes \Gamma\left(E_{i}^{\prime}\right) \rightarrow \Gamma\left(L^{2}\right)
$$

factors through $\operatorname{Sym}_{2}\left(\Gamma\left(E_{i}\right)\right) \otimes \operatorname{Sym}_{2}\left(\Gamma\left(E_{i}^{\prime}\right)\right)$, and $\varphi_{1}$ is the direct sum of the associated maps from $\operatorname{Sym}_{2}\left(\Gamma\left(E_{i}\right)\right) \otimes \operatorname{Sym}_{2}\left(\Gamma\left(E_{i}^{\prime}\right)\right)$ to $\Gamma\left(L^{2}\right)$. The map $\varphi$ is induced by $\varphi_{2}$. From ( $\left.\dagger\right)$, we see that the image of $\varphi$ is generated by the 2-minors of the matrices $\Omega\left(E_{i}, E_{i}^{\prime}\right)$.

Finally, the line bundle $L$ satisfies $N_{1}$, so the quadratic component $\left(I_{X \mid \mathbb{P} r}\right)_{2}$

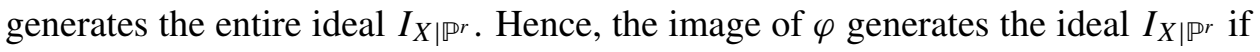
and only if $\varphi$ is surjective. Since $\varphi_{2}$ is surjective, the snake lemma (for example, [Weibel 1994, Lemma 1.3.2]) shows that the surjectivity of $\varphi$ is equivalent to the surjectivity of $\psi$.

Our main application for Lemma 3.1 focuses on a single factorization of the line bundle $L$. The proof follows the strategy in [Eisenbud et al. 1988, Section 2].

Theorem 3.2. Let $L$ be a very ample line bundle on a scheme $X$ satisfying $N_{1}$. If $L=E \otimes E^{\prime}$ for some nontrivial $E, E^{\prime} \in \operatorname{Pic}(X)$ and the conditions

(a) E has a linear presentation with respect to $E^{\prime}$,

(b) $E^{\prime}$ has a linear presentation with respect to $E$,

(c) $E^{2}$ has a linear presentation with respect to $E^{\prime}$, and

(d) both $E$ and $E^{\prime}$ satisfy $N_{0}$

hold, then the 2-minors of the matrix $\Omega\left(E, E^{\prime}\right)$ generate the homogeneous ideal of $X$ in $\mathbb{P}(\Gamma(L))$. In particular, the line bundle $L$ is determinantally presented. 
Proof. Given Lemma 3.1, it suffices to show that the map $\psi: Q_{2} \rightarrow Q_{1}$ is surjective. To accomplish this, we reinterpret both modules. Since condition (a) or (b) implies that the map $\mu_{E, E^{\prime}}: \Gamma(E) \otimes \Gamma\left(E^{\prime}\right) \rightarrow \Gamma(L)$ is surjective, we get an exact sequence

$$
\operatorname{Ker}\left(\mu_{E, E^{\prime}}\right) \otimes \Gamma(E) \otimes \Gamma\left(E^{\prime}\right) \rightarrow \operatorname{Sym}_{2}\left(\Gamma(E) \otimes \Gamma\left(E^{\prime}\right)\right) \rightarrow \operatorname{Sym}_{2}(\Gamma(L)) \rightarrow 0,
$$

so the image of $\operatorname{Ker}\left(\mu_{E, E^{\prime}}\right) \otimes \Gamma(E) \otimes \Gamma\left(E^{\prime}\right)$ generates $Q_{2}$ in $\operatorname{Sym}_{2}\left(\Gamma(E) \otimes \Gamma\left(E^{\prime}\right)\right)$. The maps $\mu_{E, E}$ and $\mu_{E^{\prime}, E^{\prime}}$ factor through $\operatorname{Sym}_{2}(\Gamma(E))$ and $\operatorname{Sym}_{2}\left(\Gamma\left(E^{\prime}\right)\right)$ and thus induce maps $\eta: \operatorname{Sym}_{2}(\Gamma(E)) \rightarrow \Gamma\left(E^{2}\right)$ and $\eta^{\prime}: \operatorname{Sym}_{2}\left(\Gamma\left(E^{\prime}\right)\right) \rightarrow \Gamma\left(E^{\prime 2}\right)$, respectively. It follows that $\varphi_{1}$ is the composition

$$
\mu_{E^{2}, E^{\prime 2}} \circ\left(\eta \otimes \eta^{\prime}\right): \operatorname{Sym}_{2}(\Gamma(E)) \otimes \operatorname{Sym}_{2}\left(\Gamma\left(E^{\prime}\right)\right) \rightarrow \Gamma\left(E^{2} \otimes E^{\prime 2}\right)=\Gamma\left(L^{2}\right) .
$$

Hence, $Q_{1}$ is the sum of the images of

$$
\operatorname{Ker}(\eta) \otimes \Gamma\left(E^{\prime}\right) \otimes \Gamma\left(E^{\prime}\right) \quad \text { and } \quad \Gamma(E) \otimes \Gamma(E) \otimes \operatorname{Ker}\left(\eta^{\prime}\right),
$$

and the pullback to $\operatorname{Sym}_{2}(\Gamma(E)) \otimes \operatorname{Sym}_{2}\left(\Gamma\left(E^{\prime}\right)\right)$ of $\operatorname{Ker}\left(\mu_{E^{2}, E^{\prime 2}}\right)$.

We now break the proof that $Q_{2}$ surjects onto $Q_{1}$ into four steps:

(i) The image of $\operatorname{Ker}\left(\mu_{E^{2}, E^{\prime}}\right) \otimes \Gamma\left(E^{\prime}\right)$ in $\Gamma\left(E^{2}\right) \otimes \Gamma\left(E^{\prime 2}\right)$ contains $\operatorname{Ker}\left(\mu_{E^{2}, E^{2}}\right)$.

(ii) The image of $\operatorname{Ker}\left(\mu_{E, E^{\prime}}\right) \otimes \Gamma(E)$ in $\Gamma\left(E^{2}\right) \otimes \Gamma\left(E^{\prime}\right)$ contains $\operatorname{Ker}\left(\mu_{E^{2}, E^{\prime}}\right)$.

(iii) The image of $\operatorname{Ker}\left(\mu_{E, E^{\prime}}\right) \otimes \Gamma(E)$ in $\operatorname{Sym}_{2}(\Gamma(E)) \otimes \Gamma\left(E^{\prime}\right)$ contains $\operatorname{Ker}(\eta) \otimes$ $\Gamma\left(E^{\prime}\right)$.

(iv) The image of $\operatorname{Ker}\left(\mu_{E, E^{\prime}}\right) \otimes \Gamma\left(E^{\prime}\right)$ in $\Gamma(E) \otimes \operatorname{Sym}_{2}\left(\Gamma\left(E^{\prime}\right)\right)$ contains $\Gamma(E) \otimes$ $\operatorname{Ker}\left(\eta^{\prime}\right)$.

By tensoring with the $\mathbb{k}$-vector space $\Gamma\left(E^{\prime}\right)$, step (ii) yields a surjective map

$$
\operatorname{Ker}\left(\mu_{E, E^{\prime}}\right) \otimes \Gamma(E) \otimes \Gamma\left(E^{\prime}\right) \rightarrow \operatorname{Ker}\left(\mu_{E^{2}, E^{\prime}}\right) \otimes \Gamma\left(E^{\prime}\right) .
$$

Combining this with step (i) shows that $\operatorname{Ker}\left(\mu_{E, E^{\prime}}\right) \otimes \Gamma(E) \otimes \Gamma\left(E^{\prime}\right) \rightarrow \operatorname{Ker}\left(\mu_{E^{2}, E^{\prime 2}}\right)$ is surjective. Again by tensoring with $\mathbb{k}$-vector space $\Gamma\left(E^{\prime}\right)$, step (iii) gives a surjective map $\operatorname{Ker}\left(\mu_{E, E^{\prime}}\right) \otimes \Gamma(E) \otimes \Gamma\left(E^{\prime}\right) \rightarrow \operatorname{Ker}(\eta) \otimes \Gamma\left(E^{\prime}\right) \otimes \Gamma\left(E^{\prime}\right)$. Similarly, step (iv) implies that the map $\operatorname{Ker}\left(\mu_{E, E^{\prime}}\right) \otimes \Gamma(E) \otimes \Gamma\left(E^{\prime}\right) \rightarrow \Gamma(E) \otimes \Gamma(E) \otimes \operatorname{Ker}\left(\eta^{\prime}\right)$ is surjective. Therefore, it is enough to establish the four steps.

For step (i), condition (c) implies that $\operatorname{Ker}\left(\mu_{E^{2}, E^{\prime}}\right)$, the span of the linear relations on $\bigoplus_{j \geqslant 0} H^{0}\left(X, E^{2} \otimes E^{j}\right)$ regarded as a $\operatorname{Sym}\left(\Gamma\left(E^{\prime}\right)\right)$-module, generates the relations in higher degrees as well. Hence, $\operatorname{Ker}\left(\mu_{E^{2}, E^{\prime}}\right) \otimes \Gamma\left(E^{\prime}\right)$ maps onto the quadratic relations that are the kernel of the composite map $\mu_{E^{2}, E^{\prime 2}} \circ\left(\mathbb{1}_{\Gamma\left(E^{2}\right)} \otimes \eta^{\prime}\right)$. Since this kernel is generated by $\Gamma\left(E^{2}\right) \otimes \operatorname{Ker}\left(\eta^{\prime}\right)$ and the pullback of $\operatorname{Ker}\left(\mu_{E^{2}, E^{\prime 2}}\right)$, Condition (d) implies that $\eta^{\prime}$ is surjective, and we have established step (i).

To complete the proof, we simultaneously establish steps (ii) and (iii); the symmetric argument yields step (iv). Condition (b) implies that $\operatorname{Ker}\left(\mu_{E, E^{\prime}}\right)$ generates 
all the relations on $\bigoplus_{j \geqslant 0} H^{0}\left(X, E^{\prime} \otimes E^{j}\right)$ regarded as a $\operatorname{Sym}(\Gamma(E))$-module. In particular, the vector space $\operatorname{Ker}\left(\mu_{E, E^{\prime}}\right) \otimes \Gamma(E)$ maps onto the quadratic relations that are the kernel of the composite map $\mu_{E^{2}, E^{\prime}} \circ\left(\eta \otimes \mathbb{1}_{\Gamma\left(E^{\prime}\right)}\right)$. This kernel is generated by $\operatorname{Ker}(\eta) \otimes \Gamma\left(E^{\prime}\right)$ and the pullback of $\operatorname{Ker}\left(\mu_{E^{2}, E^{\prime}}\right)$. Condition (d) implies that $\eta$ is surjective, so step (ii) and step (iii) follow.

As the proof indicates, Theorem 3.2 holds under a weaker version of condition (d), in that it is only necessary that $\eta$ and $\eta^{\prime}$ are surjective. Nevertheless, in all of our applications, a stronger condition is satisfied: Both $E$ and $E$ satisfy $N_{1}$.

This theorem leads to a description, given in terms of Castelnuovo-Mumford regularity, for certain determinantally presented line bundles on any projective scheme.

Corollary 3.3. Let $X$ be a connected scheme and let $B_{1}, \ldots, B_{\ell}$ be globally generated line bundles on $X$ for which there exists $\boldsymbol{w} \in \mathbb{N}^{\ell}$ such that $\boldsymbol{B}^{w}$ is ample. If $\boldsymbol{B}^{\boldsymbol{m}}$ is regular with respect to $B_{1}, \ldots, B_{\ell}$ for $\boldsymbol{m} \in \mathbb{N}^{\ell}$ and $\boldsymbol{B}^{2 \boldsymbol{m}}$ is very ample, then the line bundle $\boldsymbol{B}^{2 \boldsymbol{m}+\boldsymbol{u}}$ is determinantally presented for any $\boldsymbol{u} \in \mathbb{N}^{\ell}$.

Proof. Factor $L:=\boldsymbol{B}^{2 \boldsymbol{m}+\boldsymbol{u}}$ as $L=E \otimes E^{\prime}$ where $E:=\boldsymbol{B}^{\boldsymbol{m}}$ and $E^{\prime}:=\boldsymbol{B}^{\boldsymbol{m}+\boldsymbol{u}}$. Theorem 2.1 in [Hering et al. 2006] shows that $L, E, E^{2}$, and $E^{\prime}$ are all regular with respect to $B_{1}, \ldots, B_{\ell}$. Hence, Proposition 2.5 together with [ibid., Theorem 2.1] imply that $L, E$, and $E^{\prime}$ satisfy $N_{1}$, that $E^{\prime}$ has a linear free presentation with respect to $E$, and that both $E$ and $E^{2}$ have a linear free presentation with respect to $E^{\prime}$. Therefore, Theorem 3.2 proves that $L$ is determinantally presented.

Theorem 3.2, combined with results from Section 2, also yields a proof for our main theorem.

Proof of Theorem 1.1. Let $X$ be a connected scheme of dimension $n$ and let $B$ be a globally generated ample line bundle on $X$. Choose a positive integer $m \in \mathbb{N}$ such that $B^{m}$ is regular with respect to $B$. Lemma 2.2 implies that there exists a line bundle $E$, which we may assume is very ample, such that, for any nef line bundle $C, E \otimes C$ is regular with respect to $B$. By replacing $E$ with $E \otimes B$ if necessary, we may assume that the map $\Gamma(B) \otimes H^{0}\left(X, E \otimes B^{-1}\right) \rightarrow H^{0}(X, E)$ is surjective. Since a sufficiently ample line bundle on $X$ satisfies $N_{1}$ (combine [Inamdar 1997, Lemmas 1.1-1.3] with Fujita's vanishing theorem), we may also assume that $E \otimes C$ satisfies $N_{1}$ for any nef line bundle $C$.

Consider the line bundle $A:=E \otimes B^{2(m-1) n+1}$. If $L$ is a line bundle on $X$ such that $L \otimes A^{-1}$ is nef, then $L=A \otimes C=(E \otimes C) \otimes B^{2(m-1) n+1}$ for some nef line bundle $C$. Our choice of $E$ guarantees that, $(E \otimes C)^{j}$ is regular with respect to $B$ for all $j \geqslant 1$, and that $L$ satisfies $N_{1}$. Hence, Proposition 2.4 implies that $B^{2(m-1) n+1}$ has a linear free presentation with respect to $E \otimes C$. Proposition 2.5 together with Mumford's lemma (for example, [Lazarsfeld 2004, Theorem 1.8.5]) 
imply that both $E \otimes C$ and $(E \otimes C)^{2}$ have a linear free presentation with respect to $B^{2(m-1) n+1}$. Via Lemma 2.3 and Proposition 2.5 , we see $B^{2(m-1) n+1}$ satisfies $N_{1}$. Therefore, Theorem 3.2 proves that $L$ is determinantally presented.

\section{Effective bounds}

In this section, we give effective bounds for determinantally presented line bundles. As a basic philosophy, one can convert explicit conditions for line bundles to satisfy $N_{2}$ into effective descriptions for determinantally presented line bundles. The three subsections demonstrate this philosophy for products of projective spaces, projective Gorenstein toric varieties, and smooth varieties. Despite not developing them here, we expect similar results for general surfaces and abelian varieties following [Gallego and Purnaprajna 1999] and [Rubei 2000; Pareschi and Popa 2004], respectively.

4.1. Products of projective space. The tools from Section 3 lead to a description of the determinantally presented ample line bundles on a product of projective spaces. In contrast with [Bernardi 2008, Theorem 3.11], which proves that SegreVeronese varieties are defined by 2-minors of an appropriate hypermatrix, our classification shows that a Segre-Veronese variety is typically generated by the 2-minors of a single matrix. In particular, we recover the Segre-Veronese ideals considered in [Sullivant 2008, Section 6.2].

To study the product of projective spaces $X=\mathbb{P}^{n_{1}} \times \cdots \times \mathbb{P}^{n_{\ell}}$, we first introduce some notation. Let $R:=\mathbb{k}\left[x_{i, j}: 1 \leqslant i \leqslant \ell, 0 \leqslant j \leqslant n_{i}\right]$ be the total coordinate ring (also known as Cox ring) of $X$; this polynomial ring has the $\mathbb{Z}^{\ell}$-grading induced by $\operatorname{deg}\left(x_{i, j}\right):=\boldsymbol{e}_{i} \in \mathbb{Z}^{\ell}$. Hence, we have $R_{\boldsymbol{d}}=\Gamma\left(\mathrm{O}_{X}(\boldsymbol{d})\right)$ for all $\boldsymbol{d} \in \mathbb{Z}^{\ell}$, and a torus-invariant global section of $\mathrm{O}_{X}(d)$ is identified with a monomial $x^{w} \in R_{d}$, where $\boldsymbol{w} \in \mathbb{N}^{r}$ and $r:=\sum_{i=1}^{\ell}\left(n_{i}+1\right)$. We write $\boldsymbol{e}_{i, j}$ for the standard basis of $\mathbb{Z}^{r}$; in particular $\boldsymbol{x}^{\boldsymbol{e}_{i, j}}=x_{i, j}$.

Theorem 4.1. Let $X=\mathbb{P}^{n_{1}} \times \cdots \times \mathbb{P}^{n_{\ell}}$. An ample line bundle $\mathrm{O}_{X}(\boldsymbol{m})$ is determinantally presented if at least $\ell-2$ of the entries in the vector $\boldsymbol{m}$ are at least 2 .

When $\ell=2$, this theorem shows that all of the Segre-Veronese embeddings are determinantally presented. We note that Corollary 3.3 establishes that $O_{X}(\boldsymbol{m})$ is determinantally presented when $m_{j} \geqslant 2$ for all $1 \leqslant j \leqslant \ell$.

Proof. Since a line bundle $O_{X}(\boldsymbol{v})$ is ample (and very ample) if and only if $v_{j} \geqslant 1$ for all $1 \leqslant j \leqslant \ell$, Corollary 1.5 in [Hering et al. 2006] shows that $\bigcirc_{X}(\boldsymbol{m})$ satisfies $N_{1}$. Without loss of generality, we may assume that $m_{j} \geqslant 2$ for $1 \leqslant j \leqslant \ell-2$. Factor $\mathcal{O}_{X}(\boldsymbol{m})$ as $\mathcal{O}_{X}(\boldsymbol{m})=E \otimes E^{\prime}$, where $\boldsymbol{u}:=\boldsymbol{e}_{1}+\boldsymbol{e}_{2}+\cdots+\boldsymbol{e}_{\ell-1}=(1,1, \ldots, 1,0)$, $E:=\mathscr{O}_{X}(\boldsymbol{u})$, and $E^{\prime}:=\mathcal{O}_{X}(\boldsymbol{m}-\boldsymbol{u})$. The canonical surjection $\Gamma(E) \otimes \Gamma\left(E^{\prime}\right) \rightarrow$ $\Gamma\left(O_{X}(\boldsymbol{m})\right)$ implies that the map $\varphi_{2}$ in ( $\left.\boldsymbol{W}^{\prime}\right)$ is surjective. By Lemma 3.1, it suffices 
prove that the map $\psi: Q_{2} \rightarrow Q_{1}$ is surjective. A slight modification to the proof of [Sturmfels 1996, Lemma 4.1] shows that $Q_{1}=\operatorname{Ker}\left(\varphi_{1}\right)$ is generated by "binomial" elements in $\operatorname{Sym}_{2}(\Gamma(E)) \otimes \operatorname{Sym}_{2}\left(\Gamma\left(E^{\prime}\right)\right)$ of the form

$$
x^{a} x^{b} \otimes x^{c} x^{d}-x^{a^{\prime}} x^{b^{\prime}} \otimes x^{c^{\prime}} x^{d^{\prime}}
$$

where $\boldsymbol{x}^{\boldsymbol{a}}, \boldsymbol{x}^{\boldsymbol{b}}, \boldsymbol{x}^{\boldsymbol{a}^{\prime}}, \boldsymbol{x}^{\boldsymbol{b}^{\prime}} \in \Gamma(E), \boldsymbol{x}^{\boldsymbol{c}}, \boldsymbol{x}^{\boldsymbol{d}}, \boldsymbol{x}^{\boldsymbol{c}^{\prime}}, \boldsymbol{x}^{\boldsymbol{d}^{\prime}} \in \Gamma\left(E^{\prime}\right)$, and $\boldsymbol{a}+\boldsymbol{b}+\boldsymbol{c}+\boldsymbol{d}=$ $\boldsymbol{a}^{\prime}+\boldsymbol{b}^{\prime}+\boldsymbol{c}^{\prime}+\boldsymbol{d}^{\prime}$. Thus, the two terms in each such binomial differ by exchanging variables among the various factors. Since every such binomial element is the sum of binomials that each exchange a single pair of variables, it suffices to consider the following two cases.

In the first case, the pair of variables are exchanged between a section of $E$ and a section $E^{\prime}$. In particular, there exists some $1 \leqslant k \leqslant \ell-1$ such that the binomial element has the form

$$
\boldsymbol{x}^{\boldsymbol{a}} \boldsymbol{x}^{\boldsymbol{b}} \otimes \boldsymbol{x}^{\boldsymbol{c}} \boldsymbol{x}^{\boldsymbol{d}}-\boldsymbol{x}^{\boldsymbol{a}-\boldsymbol{e}_{k, \alpha}+\boldsymbol{e}_{k, \gamma}} \boldsymbol{x}^{\boldsymbol{b}} \otimes \boldsymbol{x}^{\boldsymbol{c}+\boldsymbol{e}_{k, \alpha}-\boldsymbol{e}_{k, \gamma}} \boldsymbol{x}^{\boldsymbol{d}},
$$

where $\boldsymbol{a}-\boldsymbol{e}_{k, \alpha}$ and $\boldsymbol{c}-\boldsymbol{e}_{k, \gamma}$ are nonnegative. This element is the image of

$$
\left(x^{a} \otimes x^{c}\right)\left(x^{b} \otimes x^{d}\right)-\left(x^{a-e_{k, \alpha}+e_{k, \gamma}} \otimes x^{c+e_{k, \alpha}-e_{k, \gamma}}\right)\left(x^{b} \otimes x^{d}\right),
$$

which lies in $Q_{2}=\operatorname{Ker}\left(\varphi_{2}\right) \subset \operatorname{Sym}_{2}\left(\Gamma(E) \otimes \Gamma\left(E^{\prime}\right)\right)$.

In the second case, we may assume that the pair of variables are exchanged between two sections of $E^{\prime}$, as exchanging variables between two sections of $E$ is analogous. More precisely, let $x_{k, \gamma}$ and $x_{k, \delta}$ for some $1 \leqslant k \leqslant \ell$ denote the exchanged variables and consider the binomial element

$$
\boldsymbol{x}^{\boldsymbol{a}} \boldsymbol{x}^{\boldsymbol{b}} \otimes \boldsymbol{x}^{\boldsymbol{c}} \boldsymbol{x}^{\boldsymbol{d}}-\boldsymbol{x}^{\boldsymbol{a}} \boldsymbol{x}^{\boldsymbol{b}} \otimes \boldsymbol{x}^{\boldsymbol{c}-\boldsymbol{e}_{k, \gamma}+\boldsymbol{e}_{k, \delta}} \boldsymbol{x}^{\boldsymbol{d}+\boldsymbol{e}_{k, \gamma}-\boldsymbol{e}_{k, \delta}}
$$

where $\boldsymbol{c}-\boldsymbol{e}_{k, \gamma}$ and $\boldsymbol{d}-\boldsymbol{e}_{k, \delta}$ are nonnegative. Since $\boldsymbol{x}^{\boldsymbol{a}} \boldsymbol{x}^{\boldsymbol{b}} \otimes \boldsymbol{x}^{\boldsymbol{c}} \boldsymbol{x}^{\boldsymbol{d}}=\boldsymbol{x}^{\boldsymbol{a}} \boldsymbol{x}^{\boldsymbol{b}} \otimes \boldsymbol{x}^{\boldsymbol{d}} \boldsymbol{x}^{\boldsymbol{c}}$ in $\operatorname{Sym}_{2}(\Gamma(E)) \otimes \operatorname{Sym}_{2}\left(\Gamma\left(E^{\prime}\right)\right)$, we may also assume that $k<\ell$. Hence, there is a variable $x_{k, \alpha}$ such that $\boldsymbol{a}-\boldsymbol{e}_{k, \alpha}$ is nonnegative and

$$
\begin{aligned}
& \boldsymbol{x}^{\boldsymbol{a}} \boldsymbol{x}^{\boldsymbol{b}} \otimes \boldsymbol{x}^{\boldsymbol{c}} \boldsymbol{x}^{\boldsymbol{d}}-\boldsymbol{x}^{\boldsymbol{a}} \boldsymbol{x}^{\boldsymbol{b}} \otimes \boldsymbol{x}^{\boldsymbol{c}-\boldsymbol{e}_{k, \gamma}+\boldsymbol{e}_{k, \delta}} \boldsymbol{x}^{\boldsymbol{d}+\boldsymbol{e}_{k, \gamma}-\boldsymbol{e}_{k, \delta}} \\
& =\boldsymbol{x}^{\boldsymbol{a}} \boldsymbol{x}^{\boldsymbol{b}} \otimes \boldsymbol{x}^{\boldsymbol{c}} \boldsymbol{x}^{\boldsymbol{d}}-\boldsymbol{x}^{\boldsymbol{a}-\boldsymbol{e}_{k, \alpha}+\boldsymbol{e}_{k, \delta}} \boldsymbol{x}^{\boldsymbol{b}} \otimes \boldsymbol{x}^{\boldsymbol{c}} \boldsymbol{x}^{\boldsymbol{d}+\boldsymbol{e}_{k, \alpha}-\boldsymbol{e}_{k, \delta}} \\
& +\boldsymbol{x}^{\boldsymbol{a}-\boldsymbol{e}_{k, \alpha}+\boldsymbol{e}_{k, \delta}} \boldsymbol{x}^{\boldsymbol{b}} \otimes \boldsymbol{x}^{\boldsymbol{c}} \boldsymbol{x}^{\boldsymbol{d}+\boldsymbol{e}_{k, \alpha}-\boldsymbol{e}_{k, \delta}}-\boldsymbol{x}^{\boldsymbol{a}-\boldsymbol{e}_{k, \alpha}+\boldsymbol{e}_{k, \gamma}} \boldsymbol{x}^{\boldsymbol{b}} \otimes \boldsymbol{x}^{\boldsymbol{c}-\boldsymbol{e}_{k, \gamma}+\boldsymbol{e}_{k, \delta}} \boldsymbol{x}^{\boldsymbol{d}+\boldsymbol{e}_{k, \alpha}-\boldsymbol{e}_{k, \delta}} \\
& +\boldsymbol{x}^{\boldsymbol{a}-\boldsymbol{e}_{k, \alpha}+\boldsymbol{e}_{k, \gamma}} \boldsymbol{x}^{\boldsymbol{b}} \otimes \boldsymbol{x}^{\boldsymbol{c}-\boldsymbol{e}_{k, \gamma}+\boldsymbol{e}_{k, \delta}} \boldsymbol{x}^{\boldsymbol{d}+\boldsymbol{e}_{k, \alpha}-\boldsymbol{e}_{k, \delta}}-\boldsymbol{x}^{\boldsymbol{a}} \boldsymbol{x}^{\boldsymbol{b}} \otimes \boldsymbol{x}^{\boldsymbol{c}-\boldsymbol{e}_{k, \gamma}+\boldsymbol{e}_{k, \delta}} \boldsymbol{x}^{\boldsymbol{d}+\boldsymbol{e}_{k, \gamma}-\boldsymbol{e}_{k, \delta}} .
\end{aligned}
$$

In other words, the binomial element under consideration is a sum of binomials in which variables are exchanged between sections of $E$ and $E^{\prime}$. Hence, the first case shows that this binomial element lies in the image of $Q_{2}$.

We conclude that $\psi$ is surjective and $\mathrm{O}_{X}(\boldsymbol{m})$ is determinantally presented. 
The next proposition shows that Theorem 4.1 is optimal when $\ell=3$. In fact, our experiments in Macaulay2 [Grayson and Stillman 2010] suggest that Theorem 4.1 is always sharp.

Proposition 4.2. If $X=\mathbb{P}^{n_{1}} \times \cdots \times \mathbb{P}^{n_{\ell}}$ with $\ell \geqslant 3$, then the ample line bundle $\mathrm{O}_{X}(\mathbf{1})$ is not determinantally presented.

Proof. Any nontrivial factorization of $\mathfrak{O}_{X}(\mathbf{1})$ has the form $E \otimes E^{\prime}$, where $E:=\mathscr{O}_{X}(\boldsymbol{u})$ for some $\boldsymbol{u} \in\{0,1\}^{\ell}$ and $E^{\prime}:=\mathscr{O}_{X}(\mathbf{1}-\boldsymbol{u})$. For a suitable choice of bases for $\Gamma\left(\mathscr{O}_{X}(\boldsymbol{u})\right)$ and $\Gamma\left(\mathscr{O}_{X}(\mathbf{1}-\boldsymbol{u})\right)$, the associated matrix $\Omega\left(\mathscr{O}_{X}(\boldsymbol{u}), \mathscr{O}_{X}(\mathbf{1}-\boldsymbol{u})\right)$ is the generic $(s \times t)$-matrix with $s:=\sum_{u_{i} \neq 0}\left(n_{i}+1\right)$ and $t:=\sum_{i=0}^{\ell}\left(n_{i}+1\right)-s$. Since the 2-minors of a generic $(s \times t)$-matrix define $\mathbb{P}^{s-1} \times \mathbb{P}^{t-1}$ in its Segre embedding, we see that $\mathrm{O}_{X}(\mathbf{1})$ is not determinantally presented when $\ell \geqslant 3$.

Example 4.3. Consider the variety $X=\mathbb{P}^{1} \times \mathbb{P}^{1} \times \mathbb{P}^{1}$ embedded in

$$
\mathbb{P}^{11}=\operatorname{Proj}\left(\mathbb{k}\left[y_{0}, \ldots, y_{11}\right]\right)
$$

by the complete linear series of $\mathcal{O}_{X}(2,1,1)$. If $R=\mathbb{k}\left[x_{1,0}, x_{1,1}, x_{2,0}, x_{2,1}, x_{3,0}, x_{3,1}\right]$ is the total coordinate ring of $X$, then the twelve monomials

$$
\left\{\begin{array}{llll}
x_{1,0}^{2} x_{2,0} x_{3,0}, & x_{1,0}^{2} x_{2,0} x_{3,1}, & x_{1,0}^{2} x_{2,1} x_{3,0}, & x_{1,0}^{2} x_{2,1} x_{3,1}, \\
x_{1,0} x_{1,1} x_{2,0} x_{3,0}, & x_{1,0} x_{1,1} x_{2,0} x_{3,1}, & x_{1,0} x_{1,1} x_{2,1} x_{3,0}, & x_{1,0} x_{1,1} x_{2,1} x_{3,1}, \\
x_{1,1}^{2} x_{2,0} x_{3,0}, & x_{1,1}^{2} x_{2,0} x_{3,1}, & x_{1,1}^{2} x_{2,1} x_{3,0}, & x_{1,1}^{2} x_{2,1} x_{3,1}
\end{array}\right\}
$$

give an ordered basis for $\Gamma\left(\mathscr{O}_{X}(2,1,1)\right)$. The homogeneous ideal $I_{X \mid \mathbb{P}^{11}}$ is the toric ideal associated to these monomials and is minimally generated by thirty three quadrics. Choosing $\left\{x_{1,0} x_{2,0}, x_{1,0} x_{2,1}, x_{1,1} x_{2,0}, x_{1,1} x_{2,1}\right\}$ as ordered basis for $\Gamma\left(\mathcal{O}_{X}(1,1,0)\right)$ and $\left\{x_{1,0} x_{3,0}, x_{1,0} x_{3,1}, x_{1,1} x_{3,0}, x_{1,1} x_{3,1}\right\}$ for $\Gamma\left(\mathcal{O}_{X}(1,0,1)\right)$, we find $\Omega\left(O_{X}(1,1,0), \mathscr{O}_{X}(1,0,1)\right)$ is

$$
\left[\begin{array}{llll}
y_{0} & y_{1} & y_{4} & y_{5} \\
y_{2} & y_{3} & y_{6} & y_{7} \\
y_{4} & y_{5} & y_{8} & y_{9} \\
y_{6} & y_{7} & y_{10} & y_{11}
\end{array}\right]
$$

and one may verify that the 2-minors of this matrix generates the ideal of $X$, so $\mathrm{O}_{X}(2,1,1)$ is determinantally presented.

However, if we consider multiple factorizations of a very ample line bundle on a product of projective spaces, then we do obtain a convenient expression of the homogeneous ideal as the 2-minors of matrices. This perspective give a conceptual explanation for both [Hà 2002, Theorem 2.6] and [Bernardi 2008, Theorem 3.11].

Proposition 4.4. If $X=\mathbb{P}^{n_{1}} \times \cdots \times \mathbb{P}^{n_{\ell}}$, then the homogeneous ideal of $X$ in $\mathbb{P}\left(\mathscr{O}_{X}(\boldsymbol{d})\right)$ is generated by the 2-minors of the matrices $\Omega\left(\mathcal{O}_{X}\left(\boldsymbol{e}_{i}\right), \mathscr{O}_{X}\left(\boldsymbol{d}-\boldsymbol{e}_{i}\right)\right)$, where $1 \leqslant i \leqslant \ell$. 
Proof. Given Theorem 4.1, we may assume that $\ell \geqslant 3$. For brevity, set $E_{i}:=\mathbb{O}_{X}\left(\boldsymbol{e}_{i}\right)$ and $E_{i}^{\prime}:=\mathscr{O}_{X}\left(\boldsymbol{d}-\boldsymbol{e}_{i}\right)$, where $1 \leqslant i \leqslant \ell$. Since $\Gamma\left(E_{i}\right) \otimes \Gamma\left(E_{i}^{\prime}\right)$ surjects onto $\Gamma\left(\mathscr{O}_{X}(\boldsymbol{d})\right)$, the map $\varphi_{2}$ in ( $\left.\Psi^{\prime}\right)$ is surjective, and it suffices to prove that the map $\psi: Q_{2} \rightarrow Q_{1}$ is surjective. By an abuse of notation, we use $\epsilon_{i}$ to denote the canonical inclusion map onto the $i$-th summand for all three of the direct sums appearing in the middle column of ( As in the proof of Theorem 4.1, $Q_{1}$ is generated by binomial elements in $\bigoplus_{k=1}^{\ell} \operatorname{Sym}_{2}\left(\Gamma\left(E_{k}\right)\right) \otimes \operatorname{Sym}_{2}\left(\Gamma\left(E_{k}^{\prime}\right)\right)$. Generators have the form

$$
\epsilon_{i}\left(x_{i, \alpha} x_{i, \beta} \otimes \boldsymbol{x}^{\boldsymbol{c}} \boldsymbol{x}^{\boldsymbol{d}}\right)-\epsilon_{j}\left(x_{j, \gamma} x_{j, \delta} \otimes \boldsymbol{x}^{\boldsymbol{a}} \boldsymbol{x}^{\boldsymbol{b}}\right),
$$

where $x_{i, \alpha}, x_{i, \beta} \in \Gamma\left(E_{i}\right), \boldsymbol{x}^{\boldsymbol{c}}, \boldsymbol{x}^{\boldsymbol{d}} \in \Gamma\left(E_{i}^{\prime}\right), x_{j, \gamma}, x_{j, \delta} \in \Gamma\left(E_{j}\right), \boldsymbol{x}^{\boldsymbol{a}}, \boldsymbol{x}^{\boldsymbol{b}} \in \Gamma\left(E_{j}^{\prime}\right)$ and $\boldsymbol{e}_{i, \alpha}+\boldsymbol{e}_{i, \beta}+\boldsymbol{c}+\boldsymbol{d}=\boldsymbol{a}+\boldsymbol{b}+\boldsymbol{e}_{j, \gamma}+\boldsymbol{e}_{j, \delta}$. We consider the following two cases.

In the first case, we have $i=j$. Since every binomial element is the sum of binomials that each exchange a single pair of variables, it suffices to consider an element of the form

$$
\epsilon_{i}\left(x_{i, \alpha} x_{i, \beta} \otimes \boldsymbol{x}^{\boldsymbol{c}} \boldsymbol{x}^{\boldsymbol{d}}-x_{i, \alpha} x_{i, \beta} \otimes \boldsymbol{x}^{\boldsymbol{c}-\boldsymbol{e}_{k, \gamma}+\boldsymbol{e}_{k, \delta}} \boldsymbol{x}^{\boldsymbol{d}+\boldsymbol{e}_{k, \gamma}-\boldsymbol{e}_{k, \delta}}\right),
$$

where $1 \leqslant k \leqslant \ell$ and both $\boldsymbol{c}-\boldsymbol{e}_{k, \gamma}$ and $\boldsymbol{d}-\boldsymbol{e}_{k, \delta}$ are nonnegative. This element is the image of

$$
\begin{array}{r}
\epsilon_{i}\left(\left(x_{i, \alpha} \otimes \boldsymbol{x}^{\boldsymbol{c}}\right)\left(x_{i, \beta} \otimes \boldsymbol{x}^{\boldsymbol{d}}\right)\right. \\
\left.-\left(x_{i, \alpha} \otimes \boldsymbol{x}^{\boldsymbol{c}-\boldsymbol{e}_{k, \gamma}+\boldsymbol{e}_{k, \delta}}\right)\left(x_{i, \beta} \otimes \boldsymbol{x}^{\boldsymbol{d}+\boldsymbol{e}_{k, \gamma}-\boldsymbol{e}_{k, \delta}}\right)\right) \\
-\epsilon_{k}\left(( x _ { k , \gamma } \otimes \boldsymbol { x } ^ { \boldsymbol { c } + \boldsymbol { e } _ { i , \alpha } - \boldsymbol { e } _ { k , \gamma } } ) \left(x_{k, \delta} \otimes \boldsymbol{x}^{\left.\boldsymbol{d}+\boldsymbol{e}_{i, \beta}-\boldsymbol{e}_{k, \delta}\right)}\right.\right. \\
\left.\quad-\left(x_{k, \delta} \otimes \boldsymbol{x}^{\boldsymbol{c}+\boldsymbol{e}_{i, \alpha}-\boldsymbol{e}_{k, \gamma}}\right)\left(x_{k, \gamma} \otimes \boldsymbol{x}^{\boldsymbol{d}+\boldsymbol{e}_{i, \beta}-\boldsymbol{e}_{k, \delta}}\right)\right),
\end{array}
$$

which lies in $Q_{2}=\operatorname{Ker}\left(\varphi_{2}\right)$.

For the second case, we have $i \neq j$. We may assume that the binomial element has the form $\epsilon_{i}\left(x_{i, \alpha} x_{i, \beta} \otimes \boldsymbol{x}^{\boldsymbol{c}} \boldsymbol{x}^{\boldsymbol{d}}\right)-\epsilon_{j}\left(x_{j, \gamma} x_{j, \delta} \otimes \boldsymbol{x}^{\boldsymbol{c}+\boldsymbol{e}_{i, \alpha}-\boldsymbol{e}_{j, \gamma}} \boldsymbol{x}^{\boldsymbol{d}+\boldsymbol{e}_{i, \beta}-\boldsymbol{e}_{j, \delta}}\right)$, where $\boldsymbol{c}-\boldsymbol{e}_{j, \gamma}$ and $\boldsymbol{d}-\boldsymbol{e}_{j, \delta}$ are nonnegative, because any additional exchanges of variables can be obtained by adding elements from the first case. This element is the image of

$$
\epsilon_{i}\left(\left(x_{i, \alpha} \otimes \boldsymbol{x}^{\boldsymbol{c}}\right)\left(x_{i, \beta} \otimes \boldsymbol{x}^{\boldsymbol{d}}\right)\right)-\epsilon_{j}\left(\left(x_{j, \gamma} \otimes \boldsymbol{x}^{\boldsymbol{c}+\boldsymbol{e}_{i, \alpha}-\boldsymbol{e}_{j, \gamma}}\right)\left(x_{j, \delta} \otimes \boldsymbol{x}^{\boldsymbol{d}+\boldsymbol{e}_{i, \beta}-\boldsymbol{e}_{j, \delta}}\right)\right),
$$

which lies in $Q_{2}=\operatorname{Ker}\left(\varphi_{2}\right)$.

Example 4.5. We consider the variety $X=\mathbb{P}^{1} \times \mathbb{P}^{1} \times \mathbb{P}^{1}$ embedded in $\mathbb{P}^{7}=$ $\operatorname{Proj}\left(\mathbb{k}\left[y_{0}, \ldots, y_{7}\right]\right)$ by the complete linear series of the line bundle $\mathcal{O}_{X}(1,1,1)$. The homogeneous ideal $I_{X \mid \mathbb{P}^{7}}$ is the toric ideal associated to the monomial list

$$
\left\{\begin{array}{llll}
x_{1,0} x_{2,0} x_{3,0}, & x_{1,0} x_{2,0} x_{3,1}, & x_{1,0} x_{2,1} x_{3,0}, & x_{1,0} x_{2,1} x_{3,1}, \\
x_{1,1} x_{2,0} x_{3,0}, & x_{1,1} x_{2,0} x_{3,1}, & x_{1,1} x_{2,1} x_{3,0}, & x_{1,1} x_{2,1} x_{3,1}
\end{array}\right\}
$$


and is minimally generated by nine quadrics. Choosing appropriate monomials for the ordered bases of the global sections, we obtain

$$
\begin{aligned}
& \Omega\left(\mathcal{O}_{X}(1,0,0), \mathscr{O}_{X}(0,1,1)\right)=\left[\begin{array}{llll}
y_{0} & y_{1} & y_{2} & y_{3} \\
y_{4} & y_{5} & y_{6} & y_{7}
\end{array}\right], \\
& \Omega\left(\mathscr{O}_{X}(0,1,0), \mathscr{O}_{X}(1,0,1)\right)=\left[\begin{array}{llll}
y_{0} & y_{1} & y_{4} & y_{5} \\
y_{2} & y_{3} & y_{6} & y_{7}
\end{array}\right] \text {, } \\
& \Omega\left(\mathcal{O}_{X}(0,0,1), \mathscr{O}_{X}(1,1,0)\right)=\left[\begin{array}{llll}
y_{0} & y_{2} & y_{4} & y_{6} \\
y_{1} & y_{3} & y_{5} & y_{7}
\end{array}\right] .
\end{aligned}
$$

It follows that $\mathfrak{O}_{X}(1,1,1)$ is not determinantally presented, but one easily verifies that the ideal $I_{X \mid \mathbb{P}^{7}}$ is generated by the 2-minors of all three matrices.

Multiple factorizations of a very ample line bundle allow one to describe a larger number of homogeneous ideals via 2 -minors. With this in mind, it would be interesting to write down the analogue of Theorem 3.2 for multiple factorizations of the line bundle.

4.2. Toric varieties. In addition to the bound given in Corollary 3.3, there is an effective bound for toric varieties involving adjoint bundles for toric varieties; see [Hering et al. 2006, Corollary 1.6]. Recall that a line bundle on a toric variety $X$ is nef if and only if it is globally generated, and the dualizing sheaf $K_{X}$ is a line bundle if and only if $X$ is Gorenstein.

Proposition 4.6. Let $X$ be a projective n-dimensional Gorenstein toric variety with dualizing sheaf $K_{X}$, and let $B_{1}, \ldots, B_{\ell}$ be the minimal generators of its nef cone $\operatorname{Nef}(X)$. Suppose that $\boldsymbol{m}, \boldsymbol{m}^{\prime} \in \mathbb{N}^{\ell}$ satisfy $\boldsymbol{B}^{\boldsymbol{m}-\boldsymbol{u}}, \boldsymbol{B}^{\boldsymbol{m}^{\prime}-\boldsymbol{u}} \in \mathfrak{B}$ for all $\boldsymbol{u} \in \mathbb{N}^{\ell}$ with $|\boldsymbol{u}| \leqslant n+1$. If $X \neq \mathbb{P}^{n}$ and $\boldsymbol{w} \in \mathbb{N}^{\ell}$, then $L=K_{X}^{2} \otimes \boldsymbol{B}^{\boldsymbol{m}+\boldsymbol{m}^{\prime}+\boldsymbol{w}}$ is determinantally presented.

Proof. Factor $L$ as $L=E \otimes E^{\prime}$, where $E:=K_{X} \otimes \boldsymbol{B}^{\boldsymbol{m}+\boldsymbol{w}}$ and $E^{\prime}:=K_{X} \otimes \boldsymbol{B}^{\boldsymbol{m}^{\prime}}$. Since $\boldsymbol{B}^{\boldsymbol{m}-(n+1) \boldsymbol{e}_{j}}, \boldsymbol{B}^{\boldsymbol{m}^{\prime}-(n+1) \boldsymbol{e}_{j}} \in \mathfrak{B}$, Corollary 0.2 in [Fujino 2003] implies that $E \otimes \boldsymbol{B}^{-\boldsymbol{e}_{j}}$ and $E^{\prime} \otimes \boldsymbol{B}^{-\boldsymbol{e}_{j}}$ belong to $\mathfrak{B}$ for all $1 \leqslant j \leqslant \ell$. For any torus-invariant curve $Y$, there is a $\boldsymbol{B}^{\boldsymbol{e}_{j}}$ such that $\boldsymbol{B}^{\boldsymbol{e}_{j}} \cdot Y>0$. Theorem 3.4 in [Mustaţă 2002] implies that $E, E^{2}$ and $E^{\prime}$ are regular with respect to $B_{1}, \ldots, B_{\ell}$. Hence, Proposition 2.5 shows that $L, E$, and $E^{\prime}$ satisfy $N_{1}$, and that $E$ has a linear free presentation with respect to $E^{\prime}$, that $E^{\prime}$ has a linear free presentation with respect to $E$, and that $E^{2}$ has a linear free presentation with respect to $E^{\prime}$. Therefore, Theorem 3.2 shows that $L$ is determinantally presented.

Proof of Theorem 1.3 for toric varieties. This is a special case of Proposition 4.6.

We give an example showing that Theorem 1.3 is not sharp for all toric varieties. 
Example 4.7. Consider the toric del Pezzo surface $X$ obtained by blowing up $\mathbb{P}^{2}$ at the three torus-fixed points. Let $R:=\mathbb{k}\left[x_{0}, \ldots, x_{5}\right]$ be the total coordinate ring of $X$. The anticanonical bundle $K_{X}^{-1}$ is very ample and corresponds to polygon

$$
P:=\operatorname{conv}\{(1,0),(1,1),(0,1),(-1,0),(-1,-1),(0,-1)\} .
$$

It is easy to see that the polygon $P$ is the smallest lattice polygon with its inner normal fan. The polygon $2 P$ contains 19 lattice points. The corresponding monomials

$$
\left\{\begin{array}{lllll}
x_{0}^{4} x_{1}^{4} x_{2}^{2} x_{5}^{2}, & x_{0}^{4} x_{1}^{3} x_{2} x_{4} x_{5}^{3}, & x_{0}^{4} x_{1}^{2} x_{4}^{2} x_{5}^{4}, & x_{0}^{3} x_{1}^{4} x_{2}^{3} x_{3} x_{5}, & x_{0}^{3} x_{1}^{3} x_{2}^{2} x_{3} x_{4} x_{5}^{2}, \\
x_{0}^{3} x_{1}^{2} x_{2} x_{3} x_{4}^{2} x_{5}^{3}, & x_{0}^{3} x_{1} x_{3} x_{4}^{3} x_{5}^{4}, & x_{0}^{2} x_{1}^{4} x_{2}^{4} x_{3}^{2}, & x_{0}^{2} x_{1}^{3} x_{2}^{3} x_{3}^{2} x_{4} x_{5}, & x_{0}^{2} x_{1}^{2} x_{2}^{2} x_{3}^{2} x_{4}^{2} x_{5}^{2}, \\
x_{0}^{2} x_{1} x_{2} x_{3}^{2} x_{4}^{3} x_{5}^{3}, & x_{0}^{2} x_{3}^{2} x_{4}^{4} x_{5}^{4}, & x_{0} x_{1}^{3} x_{2}^{4} x_{3}^{3} x_{4}, & x_{0} x_{1}^{2} x_{2}^{3} x_{3}^{3} x_{4}^{2} x_{5}, & x_{0} x_{1} x_{2}^{2} x_{3}^{3} x_{4}^{3} x_{5}^{2}, \\
x_{0} x_{2} x_{3}^{3} x_{4}^{4} x_{5}^{3}, & x_{1}^{2} x_{2}^{4} x_{3}^{4} x_{4}^{2}, & x_{1} x_{2}^{3} x_{3}^{4} x_{4}^{3} x_{5}, & x_{2}^{2} x_{3}^{4} x_{4}^{4} x_{5}^{2}
\end{array}\right\}
$$

embed $X$ into $\mathbb{P}^{18}=\operatorname{Proj}\left(\mathbb{k}\left[y_{0}, \ldots, y_{18}\right]\right)$. The homogeneous ideal $I_{X \mid \mathbb{P}^{18}}$ is the toric ideal associated to these monomials and it is minimally generated by 129 quadrics. Choosing

$$
\left\{x_{0}^{2} x_{1}^{2} x_{2} x_{5}, x_{0}^{2} x_{1} x_{4} x_{5}^{2}, x_{0} x_{1}^{2} x_{2}^{2} x_{3}, x_{0} x_{1} x_{2} x_{3} x_{4} x_{5}, x_{0} x_{3} x_{4}^{2} x_{5}^{2}, x_{1} x_{2}^{2} x_{3}^{2} x_{4}, x_{2} x_{3}^{2} x_{4}^{2} x_{5}\right\}
$$

as an ordered basis for $\Gamma\left(K_{X}^{-1}\right)$, the matrix $\Omega\left(K_{X}^{-1}, K_{X}^{-1}\right)$ is

$$
\left[\begin{array}{lllllll}
y_{0} & y_{1} & y_{3} & y_{4} & y_{5} & y_{8} & y_{9} \\
y_{1} & y_{2} & y_{4} & y_{5} & y_{6} & y_{9} & y_{10} \\
y_{3} & y_{4} & y_{7} & y_{8} & y_{9} & y_{12} & y_{13} \\
y_{4} & y_{5} & y_{8} & y_{9} & y_{10} & y_{13} & y_{14} \\
y_{5} & y_{6} & y_{9} & y_{10} & y_{11} & y_{14} & y_{15} \\
y_{8} & y_{9} & y_{12} & y_{13} & y_{14} & y_{16} & y_{17} \\
y_{9} & y_{10} & y_{13} & y_{14} & y_{15} & y_{17} & y_{18}
\end{array}\right],
$$

and its 2-minors generate $I_{X \mid \mathbb{P} 18}$. However, Theorem 1.3 only establishes that the line bundle $K_{X}^{-4}=K_{X}^{2} \otimes\left(K_{X}^{-1}\right)^{2 \cdot 2+2}$ is determinantally presented.

4.3. Smooth varieties. For smooth varieties, we also have an effective bound for adjoint bundles; see Theorem 1.3.

Proof of Theorem 1.3 for smooth varieties. Factor the line bundle $L$ as $L=E \otimes E^{\prime}$ where $E:=K_{X} \otimes A^{n+1}$ and $E^{\prime}:=K_{X} \otimes A^{j-n-1} \otimes B$. Since $j \geqslant 2 n+2$ and $E$ is nef (see [Lazarsfeld 2004, Example 1.5.35]), Proposition 2.6 implies that $L, E$, and $E^{\prime}$ satisfy $N_{1}, E$ has a linear free presentation with respect to $E^{\prime}$, that $E^{\prime}$ has a linear free presentation with respect to $E$, and that $E^{2}$ has a linear free presentation with respect to $E^{\prime}$. Thus, Theorem 3.2 shows that $L$ is determinantally presented. 
We end with an example showing that the hypotheses in Theorem 1.3 are optimal without further restrictions on the varieties under consideration.

Example 4.8. Let $X=\mathrm{Gr}(2,4)$ be the Grassmannian parametrizing all two-dimensional subspaces of the vector space $\mathbb{k}^{4}$. Let $O_{X}(1)$ denote the determinant of the universal rank 2 subbundle on $X$. The associated complete linear series determines the Plücker embedding of $X$ into $\mathbb{P}^{5}=\operatorname{Proj}\left(\mathbb{k}\left[x_{1,2}, x_{1,3}, x_{1,4}, x_{2,3}, x_{2,4}, x_{3,4}\right]\right)$. As $I_{X \mid \mathbb{P}^{5}}=\left\langle x_{1,2} x_{3,4}-x_{1,3} x_{2,4}+x_{2,3} x_{1,4}\right\rangle$, it follows that $\mathscr{O}_{X}(1)$ is not determinantally presented. On the other hand, the monomials

$$
\left\{\begin{array}{lllllll}
x_{1,2}^{2}, & x_{1,2} x_{1,3}, & x_{1,2} x_{1,4}, & x_{1,2} x_{2,3}, & x_{1,2} x_{2,4}, & x_{1,2} x_{3,4}, & x_{1,3}^{2}, \\
x_{1,3} x_{1,4}, & x_{1,3} x_{2,3}, & & x_{1,3} x_{3,4}, & x_{1,4}^{2}, & x_{1,4} x_{2,3}, & x_{1,4} x_{2,4}, \\
x_{1,4} x_{3,4}, & x_{2,3}^{2}, & x_{2,3} x_{2,4}, & x_{2,3} x_{3,4}, & x_{2,4}^{2}, & x_{2,4} x_{3,4}, & x_{3,4}^{2}
\end{array}\right\}
$$

form an ordered basis for $\Gamma\left(\mathscr{O}_{X}(2)\right)$, so the complete linear series of $\mathscr{O}_{X}(2) \mathrm{em}$ beds $X$ into $\mathbb{P}^{19}=\operatorname{Proj}\left(\mathbb{k}\left[y_{0}, \ldots, y_{19}\right]\right)$. The matrix $\Omega\left(\mathscr{O}_{X}(1), \mathscr{O}_{X}(1)\right)$ is

$$
\left[\begin{array}{cccccc}
y_{0} & y_{1} & y_{2} & y_{3} & y_{4} & y_{5} \\
y_{1} & y_{6} & y_{7} & y_{8} & y_{5}+y_{11} & y_{9} \\
y_{2} & y_{7} & y_{10} & y_{11} & y_{12} & y_{13} \\
y_{3} & y_{8} & y_{11} & y_{14} & y_{15} & y_{16} \\
y_{4} & y_{5}+y_{11} & y_{12} & y_{15} & y_{17} & y_{18} \\
y_{5} & y_{9} & y_{13} & y_{16} & y_{18} & y_{19}
\end{array}\right]
$$

and the 2-minors of this matrix generated $I_{X||^{19}}$ (indeed, this is the second Veronese of the Plücker embedding). Since $K_{X}=\mathrm{O}_{X}(-4)$ and $\mathrm{O}_{X}(2)=K_{X}^{2} \otimes \mathrm{O}_{X}(1)^{2 \cdot 4+2}$, we see that the bound in Theorem 1.3 is sharp in this case.

\section{Acknowledgements}

We thank David Eisenbud, Tony Geramita, Rob Lazarsfeld, and Pete Vermeire for helpful discussions. The computer software Macaulay2 [Grayson and Stillman 2010] was useful for generating examples. We are grateful to the referee for the careful reading.

\section{References}

[Arapura 2004] D. Arapura, "Frobenius amplitude and strong vanishing theorems for vector bundles", Duke Math. J. 121:2 (2004), 231-267. MR 2005d:14025 Zbl 1067.14018

[Bernardi 2008] A. Bernardi, "Ideals of varieties parameterized by certain symmetric tensors", $J$. Pure Appl. Algebra 212:6 (2008), 1542-1559. MR 2009c:14106 Zbl 1131.14055

[Graf v. Bothmer and Hulek 2004] H.-C. Graf v. Bothmer and K. Hulek, "Geometric syzygies of elliptic normal curves and their secant varieties", Manuscripta Math. 113:1 (2004), 35-68. MR 2006b:14009 Zbl 1053.14032 
[Buczyński and Buczyński 2010] W. Buczyński and J. Buczyński, "Secant varieties to high degree Veronese reembeddings, catalecticant matrices and smoothable Gorenstein schemes", preprint, version 2, 2010. arXiv 1012.3563v2

[Buczyński et al. 2010] J. Buczyński, A. Ginensky, and J. Landsberg, "Determinental equations for secant varieties and the Eisenbud-Koh-Stillman conjecture”, preprint, 2010. arXiv 1007.0192

[Catalano-Johnson 1996] M. L. Catalano-Johnson, "The possible dimensions of the higher secant varieties”, Amer. J. Math. 118:2 (1996), 355-361. MR 97a:14058 Zbl 0871.14043

[Catalisano et al. 2008] M. V. Catalisano, A. V. Geramita, and A. Gimigliano, "On the ideals of secant varieties to certain rational varieties", J. Algebra 319:5 (2008), 1913-1931. MR 2009g:14068 Zbl 1142.14035

[Ein and Lazarsfeld 1993] L. Ein and R. Lazarsfeld, "Syzygies and Koszul cohomology of smooth projective varieties of arbitrary dimension", Invent. Math. 111:1 (1993), 51-67. MR 93m:13006 Zbl 0814.14040

[Eisenbud 1988] D. Eisenbud, "Linear sections of determinantal varieties", Amer. J. Math. 110:3 (1988), 541-575. MR 89h:14041 Zbl 0681.14028

[Eisenbud 2005] D. Eisenbud, The geometry of syzygies, Graduate Texts in Math. 229, Springer, New York, 2005. MR 2005h:13021 Zbl 1086.14044

[Eisenbud et al. 1988] D. Eisenbud, J. Koh, and M. Stillman, "Determinantal equations for curves of high degree", Amer. J. Math. 110:3 (1988), 513-539. MR 89g:14023 Zbl 0681.14027

[Fujino 2003] O. Fujino, "Notes on toric varieties from Mori theoretic viewpoint", Tohoku Math. J. (2) 55:4 (2003), 551-564. MR 2004j:14059 Zbl 1078.14077

[Fujita 1983] T. Fujita, "Vanishing theorems for semipositive line bundles", pp. 519-528 in Algebraic geometry (Tokyo/Kyoto, 1982), Lecture Notes in Math. 1016, Springer, Berlin, 1983. MR 85g:14023 Zbl 0522.14010

[Gallego and Purnaprajna 1999] F. J. Gallego and B. P. Purnaprajna, "Syzygies of projective surfaces: an overview”, J. Ramanujan Math. Soc. 14 (1999), 65-93. MR 2000f:14019 Zbl 1058.14025

[Garcia et al. 2005] L. D. Garcia, M. Stillman, and B. Sturmfels, "Algebraic geometry of Bayesian networks", J. Symbolic Comput. 39:3-4 (2005), 331-355. MR 2006g:68242 Zbl 1126.68102

[Ginensky 2010] A. Ginensky, "A generalization of the Clifford index and determinantal equations for curves and their secant varieties", preprint, 2010. arXiv 1002.2023

[Grayson and Stillman 2010] D. Grayson and M. Stillman, Macaulay2, a software system for research in algebraic geometry, 2010, available at http://www.math.uiuc.edu/Macaulay2/.

[Green 1984a] M. L. Green, "Koszul cohomology and the geometry of projective varieties", J. Differential Geom. 19:1 (1984), 125-171. MR 85e:14022 Zbl 0559.14008

[Green 1984b] M. L. Green, "Koszul cohomology and the geometry of projective varieties, II", $J$. Differential Geom. 20:1 (1984), 279-289. MR 86j:14011 Zbl 0559.14009

[Green 1989] M. L. Green, “Koszul cohomology and geometry”, pp. 177-200 in Lectures on Riemann surfaces (Trieste, 1987), edited by M. Raynaud and T. Shioda, World Sci. Publ., Teaneck, NJ, 1989. MR 91k:14012 Zbl 0800.14004

[Green and Lazarsfeld 1985] M. Green and R. Lazarsfeld, "On the projective normality of complete linear series on an algebraic curve", Invent. Math. 83:1 (1985), 73-90. MR 87g:14022 Zbl 0594.14010

[Hà 2002] H. T. Hà, "Box-shaped matrices and the defining ideal of certain blowup surfaces", $J$.

Pure Appl. Algebra 167:2-3 (2002), 203-224. MR 2002h:13020 Zbl 1044.13004 
[Hering et al. 2006] M. Hering, H. Schenck, and G. G. Smith, "Syzygies, multigraded regularity and toric varieties", Compos. Math. 142:6 (2006), 1499-1506. MR 2007k:13025 Zbl 1111.14052

[Inamdar 1997] S. P. Inamdar, "On syzygies of projective varieties”, Pacific J. Math. 177:1 (1997), 71-76. MR 98a:14010 Zbl 0898.14015

[Lazarsfeld 1989] R. Lazarsfeld, "A sampling of vector bundle techniques in the study of linear series", pp. 500-559 in Lectures on Riemann surfaces (Trieste, 1987), edited by M. Raynaud and T. Shioda, World Sci. Publ., Teaneck, NJ, 1989. MR 92f:14006 Zbl 0800.14003

[Lazarsfeld 2004] R. Lazarsfeld, Positivity in algebraic geometry, I: Classical setting: line bundles and linear series, Ergebnisse der Mathematik und ihrer Grenzgebiete (3) 48, Springer, Berlin, 2004. MR 2005k:14001a Zbl 1093.14501

[Maclagan and Smith 2004] D. Maclagan and G. G. Smith, "Multigraded Castelnuovo-Mumford regularity”, J. Reine Angew. Math. 571 (2004), 179-212. MR 2005g:13027 Zbl 1062.13004

[Mumford 1970] D. Mumford, "Varieties defined by quadratic equations", pp. 29-100 in Questions on Algebraic Varieties (C.I.M.E., III) (Ciclo, Varenna, 1969), Edizioni Cremonese, Rome, 1970. MR 44 \#209 Zbl 0198.25801

[Mustaţă 2002] M. Mustaţă, "Vanishing theorems on toric varieties", Tohoku Math. J. (2) 54:3 (2002), 451-470. MR 2003e:14013 Zbl 1092.14064

[Pareschi and Popa 2004] G. Pareschi and M. Popa, "Regularity on abelian varieties, II: Basic results on linear series and defining equations", J. Algebraic Geom. 13 (2004), 167-193. MR 2005a:14059 Zbl 1073.14061

[Ravi 1994] M. S. Ravi, "Determinantal equations for secant varieties of curves", Comm. Algebra 22:8 (1994), 3103-3106. MR 95c:14029 Zbl 0809.14038

[Room 1938] T. G. Room, The Geometry of Determinantal Loci, Cambridge University Press, 1938. Zbl 0020.05402 JFM 64.0693.04

[Rubei 2000] E. Rubei, “On syzygies of abelian varieties”, Trans. Amer. Math. Soc. 352:6 (2000), 2569-2579. MR 2000j:14071 Zbl 0967.14013

[Sturmfels 1996] B. Sturmfels, Gröbner bases and convex polytopes, University Lecture Series 8, American Mathematical Society, Providence, RI, 1996. MR 97b:13034 Zbl 0856.13020

[Sturmfels and Sullivant 2006] B. Sturmfels and S. Sullivant, "Combinatorial secant varieties", Pure Appl. Math. Q. 2:3 (2006), 867-891. MR 2007h:14082 Zbl 1107.14045

[Sullivant 2008] S. Sullivant, "Combinatorial symbolic powers", J. Algebra 319:1 (2008), 115-142. MR 2009c:13005 Zbl 1133.13027

[Weibel 1994] C. A. Weibel, An introduction to homological algebra, Cambridge Studies in Advanced Mathematics 38, Cambridge University Press, 1994. MR 95f:18001 Zbl 0797.18001

Communicated by Kei-Ichi Watanabe

Received 2010-05-20 Revised 2011-05-31 Accepted 2011-06-30

jsidman@mtholyoke.edu Department of Mathematics and Statistics,

Mount Holyoke College, 415A Clapp Lab, South Hadley, MA 01075, United States http://www.mtholyoke.edu/ jsidman/

ggsmith@mast.queensu.ca Department of Mathematics and Statistics, Queen's University, 512 Jeffery Hall, University Avenue, Kingston, ON K7L 3N6, Canada http://www. mast.queensu.ca/ ggsmith/ 


\section{Algebra \& Number Theory}

msp.berkeley.edu/ant

\section{EDITORS}

MANAGING EDITOR

Bjorn Poonen

Massachusetts Institute of Technology

Cambridge, USA

\author{
EDITORIAL BOARD CHAIR \\ David Eisenbud \\ University of California \\ Berkeley, USA
}

\section{BOARD OF EDITORS}

Georgia Benkart

Dave Benson

Richard E. Borcherds

John H. Coates

J-L. Colliot-Thélène

Brian D. Conrad

Hélène Esnault

Hubert Flenner

Edward Frenkel

Andrew Granville

Joseph Gubeladze

Ehud Hrushovski

Craig Huneke

Mikhail Kapranov

Yujiro Kawamata

János Kollár

Yuri Manin

Barry Mazur

Philippe Michel

Susan Montgomery
University of Wisconsin, Madison, USA

University of Aberdeen, Scotland

University of California, Berkeley, USA

University of Cambridge, UK

CNRS, Université Paris-Sud, France

University of Michigan, USA

Universität Duisburg-Essen, Germany

Ruhr-Universität, Germany

University of California, Berkeley, USA

Université de Montréal, Canada

San Francisco State University, USA

Hebrew University, Israel

University of Kansas, USA

Yale University, USA

University of Tokyo, Japan

Princeton University, USA

Northwestern University, USA

Harvard University, USA

École Polytechnique Fédérale de Lausanne

University of Southern California, USA
Shigefumi Mori

Raman Parimala

Jonathan Pila

Victor Reiner

Karl Rubin

Peter Sarnak

Joseph H. Silverman

Michael Singer

Ronald Solomon

Vasudevan Srinivas

J. Toby Stafford

Bernd Sturmfels

Richard Taylor

Ravi Vakil

Michel van den Bergh

Marie-France Vignéras

Kei-Ichi Watanabe

Andrei Zelevinsky

Efim Zelmanov
RIMS, Kyoto University, Japan

Emory University, USA

University of Oxford, UK

University of Minnesota, USA

University of California, Irvine, USA

Princeton University, USA

Brown University, USA

North Carolina State University, USA

Ohio State University, USA

Tata Inst. of Fund. Research, India

University of Michigan, USA

University of California, Berkeley, USA

Harvard University, USA

Stanford University, USA

Hasselt University, Belgium

Université Paris VII, France

Nihon University, Japan

Northeastern University, USA

University of California, San Diego, USA

\section{PRODUCTION}

contact@msp.org

Silvio Levy, Scientific Editor

See inside back cover or www.jant.org for submission instructions.

The subscription price for 2011 is US \$150/year for the electronic version, and \$210/year (+\$35 shipping outside the US) for print and electronic. Subscriptions, requests for back issues from the last three years and changes of subscribers address should be sent to Mathematical Sciences Publishers, Department of Mathematics, University of California, Berkeley, CA 94720-3840, USA.

Algebra \& Number Theory (ISSN 1937-0652) at Mathematical Sciences Publishers, Department of Mathematics, University of California, Berkeley, CA 94720-3840 is published continuously online. Periodical rate postage paid at Berkeley, CA 94704, and additional mailing offices.

ANT peer review and production are managed by EditFLOW ${ }^{\circledR}$ from Mathematical Sciences Publishers.

PUBLISHED BY

mathematical sciences publishers

http://msp.org/

A NON-PROFIT CORPORATION

Typeset in IAT $_{\mathrm{E}} \mathrm{X}$

Copyright @2011 by Mathematical Sciences Publishers 


\section{Algebra \& Number Theory}

\section{Volume $5 \quad$ No. $8 \quad 2011$}

The behavior of Hecke $L$-functions of real quadratic fields at $s=0$ BYUNGHEUP JUN and JUNGYUN LEE

The Picard group of a $K 3$ surface and its reduction modulo $p$

ANDREAS-STEPHAN ELSENHANS and JÖRG JAHNEL

Linear determinantal equations for all projective schemes JESSICA SIDMAN and GREGORY G. SMITH

Involutions, weights and $p$-local structure

GEOFFREY R. ROBINSON

Parametrizing quartic algebras over an arbitrary base

MELANIE MatchetT WoOD

Coleman maps and the $p$-adic regulator

ANTONIO LEI, DAVID LOEFFLER and SARAH LIVIA ZERBES

Conjecture de Shafarevitch effective pour les revêtements cycliques 